TRANSACTIONS OF THE

AMERICAN MATHEMATICAL SOCIETY

Volume 363, Number 5, May 2011, Pages 2265-2294

S 0002-9947(2010)04820-2

Article electronically published on December 2, 2010

\title{
EVOLUTION OF CONVEX LENS-SHAPED NETWORKS UNDER THE CURVE SHORTENING FLOW
}

\author{
OLIVER C. SCHNÜRER, ABDERRAHIM AZOUANI, MARC GEORGI, JULIETTE HELL, \\ NIHAR JANGLE, AMOS KOELLER, TOBIAS MARXEN, SANDRA RITTHALER, \\ MARIEL SÁEZ, FELIX SCHULZE, AND BRIAN SMITH
}

\begin{abstract}
We consider convex symmetric lens-shaped networks in $\mathbb{R}^{2}$ that evolve under the curve shortening flow. We show that the enclosed convex domain shrinks to a point in finite time. Furthermore, after appropriate rescaling the evolving networks converge to a self-similarly shrinking network, which we prove to be unique in an appropriate class. We also include a classification result for some self-similarly shrinking networks.
\end{abstract}

\section{INTRODUCTION}

The evolution of networks under the curve shortening flow was studied by Carlo Mantegazza, Matteo Novaga, and Vincenzo Tortorelli [13. In their paper they investigated the case where the underlying graph consists of three lines having one point in common. In that context they proved short-time existence, long-time existence and, under extra conditions, convergence to a minimizing configuration. However, their methods allowed them to study only tree networks. In this paper we focus on the evolution of graphs with closed loops. We consider graphs with special kinds of loops, namely symmetric lens-shaped networks.

A convex lens-shaped network $M_{0} \subset \mathbb{R}^{2}$ consists of two smooth convex arcs and two half-lines arranged as in Figure 1 .

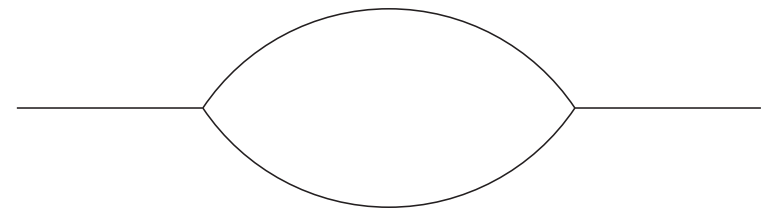

FIGURE 1. Lens-shaped network

As in [13, we assume that the curves meet at the triple points at an angle of $120^{\circ}$. We also impose an extra symmetry assumption: we consider networks that

Received by the editors April 29, 2008.

2010 Mathematics Subject Classification. Primary 53C44, 35B40.

Key words and phrases. Mean curvature flow, networks, triple junctions.

Several authors are members of and were supported by SFB 647/B3 "Raum - Zeit - Materie: Singularity structure, long-time behavior and dynamics of solutions of non-linear evolution equations". The ninth author was supported by the grant "Proyecto Fondecyt de Iniciación 11070025". 
have reflection symmetry about the $x^{1}$-axis. For precise definitions we refer the reader to Section 2 ,

In order to define the evolution equation of such a network we consider $G$ to be an abstract graph homeomorphic to $M_{0}$ consisting of two bounded intervals and two half-lines. Let $F_{0}: G \rightarrow M_{0}$ be a homeomorphism, which restricted to a closed edge is a diffeomorphism. Now consider a family $(F(\cdot, t))_{t \in I}$ of mappings $F(\cdot, t): G \rightarrow \mathbb{R}^{2}$, where each $M_{t}:=F(G, t)$ is a convex lens-shaped network as described above and $I$ denotes a generic interval. Then the family $\left(M_{t}\right)_{0 \leq t<T}$ is said to evolve under the curve shortening flow if away from the vertices it satisfies

$$
\left(\frac{d}{d t} F\right)^{\perp}=\vec{\kappa}
$$

where $\perp$ denotes the orthogonal projection on the normal space and $\vec{\kappa}$ denotes the curvature vector. Up to tangential diffeomorphisms, (1.1) is equivalent to $\frac{d}{d t} F=$ $-\kappa \nu$. Let $\Omega_{t}$ denote the bounded component of $\mathbb{R}^{2} \backslash M_{t}$.

We prove that $M_{t}$ shrinks under the curve shortening flow (1.1) to a straight line. More precisely,

Theorem 1.1. Let $M_{0}$ be a convex lens-shaped network. Then there exists a family $M_{t}:=F(G, t)$ of convex lens-shaped networks as above that evolves under the curve shortening flow (1.1). This family is smooth for $t>0$ and spatially $C^{2}$ up to $t=0$. Moreover, it exists for $t \in[0, T)$, where $T=\frac{3\left|\Omega_{0}\right|}{4 \pi}$, and, as $t \nearrow T$, the networks $M_{t}$ converge in the Hausdorff distance to the $x^{1}$-axis.

This result is analogous to the one proved by Michael Gage and Richard Hamilton [8] for convex curves embedded in $\mathbb{R}^{2}$. They showed that such curves shrink to a point in finite time. Furthermore, after appropriate rescaling, these curves converge to a circle. Notice that circles shrinking homothetically at an appropriate rate are self-similar solutions to (1.1). Moreover, in 11, Uwe Abresch and Joel Langer proved these circles are the unique embedded self-similarly shrinking solutions to curve shortening flow in the plane.

Similarly, in order to analyze the blow-up profile of shrinking lenses, we consider families of networks $\left(M_{t}\right)_{t \in I}$ that shrink self-similarly. That is, for every $t_{1}, t_{2} \in I$, the network $M_{t_{1}}$ is the image of $M_{t_{2}}$ under a homothety. The existence of such networks was studied numerically by William Mullins [14. We prove the following classification theorem:

Theorem 1.2. There exists a unique self-similarly shrinking family of symmetric lens-shaped networks $\left(N_{t}\right)_{t \in(-\infty, 0)}$ solving (1.1) such that as $t \nearrow 0$ the networks $N_{t}$ converge to the $x^{1}$-axis in the Hausdorff distance. Moreover, all networks $N_{t}$ are symmetric with respect to the $x^{2}$-axis.

As in $[8$, this enables us to describe the shape of the networks in Theorem 1.1 more closely as $t \nearrow T$ :

Theorem 1.3. Let $\left(M_{t}\right)_{t \in[0, T)}$ be a family of networks as in Theorem 1.1. Then there exists a unique $x_{0}$ such that $\left\{x_{0}\right\}=\bigcap_{[0, T)} \Omega_{t}$ and the rescaled networks converge smoothly to a network that contracts self-similarly:

$$
(2(T-t))^{-1 / 2} \cdot\left(M_{t}-x_{0}\right) \rightarrow N_{\left(-\frac{1}{2}\right)},
$$

where $N_{t}$ is as in Theorem 1.2 . 
For the sake of completeness, we also include a complete classification of a certain class of homothetically shrinking networks. Namely, we consider networks that have the same topology as the lens-shaped ones. We prove that, besides the family described in Theorem 1.2, there exists precisely one (up to rotations and reflections) such network. It is depicted as in Figure 2.

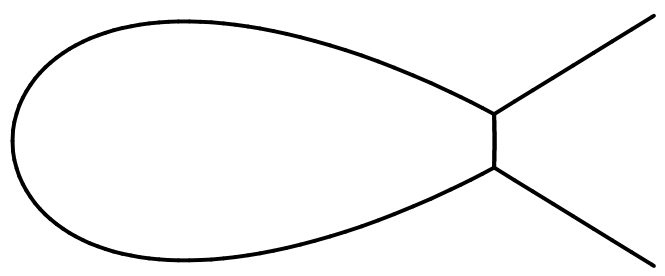

FIGURE 2. Homothetically shrinking fish-shaped network

We organize the paper as follows: In Section 2 we establish notation, rewrite the equation as a free boundary value problem and prove short-time existence. Theorem 1.1 is proved in Section 3 , that is, we show long-time existence up to a time $T$ such that $M_{t}$ converges to a line. In Section 4 we carry out a blow-up analysis that proves Theorem 1.3. The existence and uniqueness of self-similar shrinking lenses is studied in Section 5. Homothetically shrinking fish-shaped networks are considered in Appendix A

Finally, we would like to recall that the result of Michael Gage and Richard Hamilton was later extended by Matthew Grayson, who proved that embedded closed curves become convex in finite time 9. Unfortunately, an analogous extension for our problem is not included in this paper. However, this might be an interesting direction to explore in the future.

This paper was written in a research seminar organized by Oliver Schnürer at the Free University of Berlin in 2006 and 2007.

\section{Notation}

A symmetric lens-shaped network $M \subset \mathbb{R}^{2}$ is determined by a smooth curve $\gamma:[-1,1] \rightarrow \mathbb{R}^{2}$, which is regular up to the boundary, and such that the following conditions hold:

(i) $\gamma((-1,1)) \subset\left\{\left(x^{1}, x^{2}\right) \in \mathbb{R}^{2}: x^{2}>0\right\}$,

(ii) $\gamma(-1), \gamma(1) \in\left\{\left(x^{1}, x^{2}\right) \in \mathbb{R}^{2}: x^{2}=0\right\}$ and $x^{1}(\gamma(-1))<x^{1}(\gamma(1))$,

(iii) at the endpoints, the curve and the $x^{1}$-axis enclose an angle of $60^{\circ}$; more precisely,

$$
\frac{\gamma^{\prime}(-1)}{\left|\gamma^{\prime}(-1)\right|}=\left(\frac{1}{2}, \frac{\sqrt{3}}{2}\right), \quad \frac{\gamma^{\prime}(1)}{\left|\gamma^{\prime}(1)\right|}=\left(\frac{1}{2},-\frac{\sqrt{3}}{2}\right) .
$$

Then the complete symmetric lens-shaped network $M$ is given by four curves: $\gamma_{1}, \gamma_{2}, \gamma_{3}, \gamma_{4}$, where $\gamma_{1}$ is the curve $\gamma$ described above, $\gamma_{2}$ is the reflection of $\gamma_{1}$ about the $x^{1}$-axis, and $\gamma_{3}, \gamma_{4}$ are the half-lines contained in the $x^{1}$-axis connecting $\gamma_{1}(-1)$ and $\gamma_{1}(1)$ to $-\infty$ and $+\infty$, respectively. Hence, we can always identify the network $M$ with the curve $\gamma:=\gamma_{1}$. Notice that the network is convex (resp. strictly convex) if the curvature of $\gamma$ is non-negative (resp. strictly positive) with an appropriate sign convention. It is easy to verify that in the interior the family of 
symmetric lens-shaped networks $\left(\gamma_{t}\right)_{0 \leq t<T}$ evolves according to (1.1) if it satisfies the evolution equation

$$
\left\langle\dot{\gamma}_{t}, \nu\right\rangle=\frac{\left\langle\left(\gamma_{t}\right)_{x x}, \nu\right\rangle}{\left|\left(\gamma_{t}\right)_{x}\right|^{2}},
$$

where $\nu$ is a choice of unit normal vector to $\gamma_{t}$. Here and in what follows a dot will always denote the derivative with respect to time and a subscript $x$ the derivative with respect to the spatial parameter.

For such a family of networks, we define $a(t), b(t) \in \mathbb{R}, a(t)<b(t)$, for $t \in[0, T)$ by $\gamma_{t}(-1)=(a(t), 0)$ and $\gamma_{t}(1)=(b(t), 0)$. Furthermore, let $\Omega_{t}$ be the open bounded component of $\mathbb{R}^{2} \backslash M_{t}$ and $\left|\Omega_{t}\right|$ its 2-dimensional Lebesgue measure. The length of the network is defined by $L_{t}:=2 \cdot \operatorname{length}\left(\gamma_{t}\right)$.

Suppose that $\gamma_{t}([-1,1])=\left.\operatorname{graph} u(\cdot, t)\right|_{[a(t), b(t)]}$ for a function $u: \mathbb{R} \times[0, T) \rightarrow$ $[0, \infty)$. Then we call the corresponding network graphical. Notice that for times when a network is convex, it is also graphical. Moreover, the function $u$ is concave in $[a(t), b(t)]$, and the network remains graphical for some time. In this situation the problem can be reformulated as follows:

A continuous function $u: \mathbb{R} \times[0, T) \rightarrow \mathbb{R}$ determines a solution to (1.1) if there exist $a:[0, T) \rightarrow \mathbb{R}$ and $b:[0, T) \rightarrow \mathbb{R}$ with $a(t)<b(t)$ for all $t \in[0, T)$ and

$$
\begin{cases}\dot{u}=\frac{u_{x x}}{1+u_{x}^{2}} & \text { in the interior of } \bigcup_{t \in[0, T)}(a(t), b(t)) \times\{t\}, \\ u(x, t)=0 & \text { for } x \in(-\infty, a(t)] \cup[b(t), \infty) \text { and } t \in[0, T), \\ u_{x}(a(\cdot), \cdot)=\tan \frac{\pi}{3}=\sqrt{3} & \text { in }[0, T), \\ u_{x}(b(\cdot), \cdot)=-\tan \frac{\pi}{3}=-\sqrt{3} & \text { in }[0, T), \\ u(\cdot, 0)=u_{0} & \text { in } \mathbb{R},\end{cases}
$$

where $u_{0}: \mathbb{R} \rightarrow \mathbb{R}$ is such that

$$
\left\{\begin{array}{l}
u_{0}=0 \\
\left(u_{0}\right)_{x}(a(0))=\tan \frac{\pi}{3}=\sqrt{3}, \\
\left(u_{0}\right)_{x}(b(0))=-\tan \frac{\pi}{3}=-\sqrt{3} .
\end{array} \text { in }(-\infty, a(0)] \cup[b(0), \infty),\right.
$$

Here expressions such as $u_{x}(a)$ have to be considered as the limits from above, whereas expressions such as $u_{x}(b)$ are limits from below. The solutions considered are in $C^{2 ; 1}$ and, hence, we require that $u_{0} \in C^{2}([a(0), b(0)])$. Furthermore, we choose $u_{0}$ such that graph $u_{0} \cup \operatorname{graph}\left(-u_{0}\right)=M_{0}$.

Under this formulation it is easy to see that the maximum principle implies $u(x, t)>0$ for $a(t)<x<b(t)$ and that any solution to (1.1) is unique in the class of lens-shaped networks.

Short-time existence for (1.1), and thus also for (2.1), follows from [13, Theorem 3.1] by introducing artificial boundary points on the half-lines. Furthermore, estimates in 13 imply that solutions are in $C^{2 ; 1}$. Summarizing we have

Theorem 2.1 ([13)). For any smooth symmetric lens-shaped network, (1.1) has a solution of class $C^{2 ; 1}$ up to the free boundary on a short time interval.

We finish this section by writing the relevant geometric quantities in terms of the function $u$. We also recall the definition of the support function, which will be used in Section 5 . 
An easy computation shows that the upwards pointing unit normal of $\gamma_{t}=$ $\left.\operatorname{graph} u(\cdot, t)\right|_{[a(t), b(t)]}$ is

$$
\nu=\frac{\left(-u_{x}, 1\right)}{\sqrt{1+u_{x}^{2}}}
$$

The curvature is given by

$$
\kappa=\frac{-u_{x x}}{\left(1+u_{x}^{2}\right)^{3 / 2}}
$$

and as usual the curvature vector is given by $\vec{\kappa}=-\kappa \nu$. We define $\nu$ along the $\operatorname{graph}\left(-\left.u(\cdot, t)\right|_{[a(t), b(t)]}\right)$ as the reflection of the normal vector $\nu$ from above. On $\left\{x^{2}<0\right\}$ we define $\kappa$ such that $\kappa \geq 0$ for a convex lens.

We briefly recall that for a curve $\alpha: I \rightarrow \mathbb{R}^{2}$, the support function $S: \nu(\alpha(I)) \rightarrow$ $\mathbb{R}$ is defined by $S(p):=\left\langle\alpha\left(\nu^{-1}(p)\right), p\right\rangle$. More details and properties of the support function can be found in Section 5 .

\section{LONG-TIME EXISTENCE AND CONVERGENCE TO A POINT}

In this section we prove long-time existence and convergence to a point. We do this via a series of lemmata. The main ingredients are gradient estimates, a formula for the rate of decrease of the volume and curvature bounds. These last estimates are proven using a modified intrinsic-extrinsic distance ratio, that was introduced by Gerhard Huisken in [12.

Note that for the first two lemmata we do not have to assume that our networks are convex.

Lemma 3.1 (Solutions stay graphical). Let $M_{0}$ be a graphical symmetric lensshaped network. If (1.1) has a solution on a time interval $[0, T)$, then $M_{t}$ is graphical for all $t \in[0, T)$. In fact, we have for $u$ as in (2.1) the estimate

$$
\left|u_{x}\right| \leq \max \left\{\sqrt{3}, \sup _{x \in(a(0), b(0))}\left|u_{x}(x, 0)\right|\right\} .
$$

Proof. For a smooth network, being graphical means that $\left|u_{x}\right|<\infty$. Notice that for a smooth evolution of networks this condition is preserved for short times. Moreover, in order to prove the lemma it suffices to consider (2.1) instead of (1.1) and to show that $t \mapsto \max _{x \in[a(t), b(t)]}\left|u_{x}(x, t)\right|$ is non-increasing.

We employ the maximum principle on the domains where $u>0$, that is, for each $t$ we take $x \in(a(t), b(t))$. On $\{(a(t), t): t \in[0, T)\} \cup\{(b(t), t): t \in[0, T)\}$, we have $\left|u_{x}\right|=\sqrt{3}$. It is easy to compute that the evolution equation for $u_{x}$ is given by

$$
\frac{d}{d t}\left(u_{x}\right)=\frac{1}{1+u_{x}^{2}}\left(u_{x}\right)_{x x}-\frac{2 u_{x} u_{x x}}{\left(1+u_{x}^{2}\right)^{2}}\left(u_{x}\right)_{x} .
$$

Hence, according to the maximum principle, $\left|u_{x}\right|$ cannot attain a new maximum in the interior of $\bigcup_{t \in(0, T)}(a(t), b(t)) \times\{t\}$.

Lemma 3.2 (Evolution of the enclosed volume). Let $\left(M_{t}\right)_{t \in[0, T)}$ be a family of symmetric networks solving (1.1). Then the enclosed volume fulfills

$$
\frac{d}{d t}\left|\Omega_{t}\right|=-\frac{4 \pi}{3} .
$$


Proof. We give a proof only for graphical networks. In particular, our proof shows that there is no contribution to the change of enclosed area from the triple points. Near the triple points, a smooth symmetric network is always graphical. Thus, the general result can be obtained similarly as for closed curves [8].

For a graphical network, we have

$$
\left|\Omega_{t}\right|=2 \int_{a(t)}^{b(t)} u(x, t) d x .
$$

Differentiating yields

$$
\begin{aligned}
\frac{d}{d t}\left|\Omega_{t}\right| & =2 \underbrace{u(b(t), t)}_{=0} \dot{b}(t)-2 \underbrace{u(a(t), t)}_{=0} \dot{a}(t)+2 \int_{a(t)}^{b(t)} \dot{u}(x, t) d x \\
& =2 \int_{a(t)}^{b(t)} \frac{u_{x x}}{1+u_{x}^{2}}(x, t) d x=2 \arctan (\underbrace{u_{x}(b(t))}_{=-\sqrt{3}})-2 \arctan (\underbrace{u_{x}(a(t))}_{=\sqrt{3}})=-\frac{4 \pi}{3} .
\end{aligned}
$$

The lemma follows.

Note that this lemma also implies the following: If $\Omega_{t}$ shrinks to a point at time $T$ or, more generally, $\bigcap_{t \in[0, T)} \Omega_{t}$ is a set of measure zero, then $T=\frac{3 \cdot\left|\Omega_{0}\right|}{4 \pi}$.

Lemma 3.3 (Convexity is preserved). A convex network evolving (smoothly) according to (2.1) stays convex. More precisely, a non-negative lower bound on the curvatures (in the non-flat part of the network) is preserved during the evolution.

Proof. Let us first assume that the curvature of graph $u(\cdot, 0)$ is uniformly bounded below in $(a(0), b(0))$ by $\lambda>0$ (that is, the network is strictly convex).

A simple computation in $[8$ ] shows that the evolution equation for the curvature $\kappa$ is

$$
\frac{d}{d t} \kappa=\Delta \kappa+\kappa^{3}
$$

where $\Delta \kappa$ denotes the Laplace-Beltrami operator applied to $\kappa$.

Let

$$
k(t):=\inf _{\substack{\tau \in[0, t] \\ x \in[a(t), b(t)]}} \kappa(x, \tau) .
$$

Note that $k(t)$ is monotonically decreasing, Lipschitz continuous and hence differentiable for almost every $t$. Moreover, the evolution equation for $\kappa$ and the maximum principle imply that at times that $k(t)$ is decreasing and differentiable, the minimum has to be attained at the boundary and the derivative of $k(t)$ equals $\frac{d}{d t} \kappa(a(t), t)$ or $\frac{d}{d t} \kappa(b(t), t)$.

Fix $0<\varepsilon<\lambda$ arbitrarily and suppose that there is a $t_{1}$ such that $k\left(t_{1}\right)=\lambda-\varepsilon$. Assume that $t_{1}$ is minimal with that property. Hence there exists a time $t_{0}$ with $0<t_{0}<t_{1}$ such that $\frac{d}{d t} k\left(t_{0}\right)<0$. Without loss of generality we may assume that $\frac{d}{d t} k\left(t_{0}\right)=\frac{d}{d t} \kappa\left(a\left(t_{0}\right), t_{0}\right)$ and get

$$
\begin{aligned}
\left.\frac{d}{d t} \kappa(a(t), t)\right|_{t=t_{0}} & <0, \\
\kappa\left(a\left(t_{0}\right), t_{0}\right) & =\lambda-\tilde{\varepsilon}, \quad 0<\tilde{\varepsilon}<\varepsilon,
\end{aligned}
$$


and

$$
\kappa\left(a\left(t_{0}\right), t_{0}\right) \leq \inf _{\substack{t \in\left[0, t_{0}\right] \\ a(t) \leq x \leq b(t)}} \kappa(x, t)
$$

By rotating our network $90^{\circ}$ we can describe it near the triple point $(a(t), 0)$ (in the original coordinate system) as graph $\left.\tilde{u}\right|_{[0, \delta) \times[0, \tau)}$ for some $\delta>0$ and $\tau>t_{0}$. Thus $\tilde{u}$ solves

$$
\begin{cases}\dot{\tilde{u}}=\frac{\tilde{u}_{x x}}{1+\tilde{u}_{x}^{2}} & \text { in }[0, \delta) \times[0, \tau), \\ \tilde{u}_{x}(0, t)=-\frac{1}{\sqrt{3}} & \text { in }[0, \tau)\end{cases}
$$

Now we construct a barrier for $\tilde{u}$ using the downwards translating grim reaper, given by

$$
\operatorname{graph}\left(\left(-\frac{\pi}{2}, \frac{\pi}{2}\right) \ni x \mapsto \log \cos x\right) .
$$

First notice that by rescaling and translating the grim reaper it is possible to get a $\zeta>0$ and a function $\Gamma:[0, \zeta) \times \mathbb{R} \rightarrow \mathbb{R}$ that satisfies

$$
\begin{cases}\dot{\Gamma}=\frac{\Gamma_{x x}}{1+\Gamma_{x}^{2}} & \text { in }[0, \zeta) \times \mathbb{R} \\ \Gamma_{x}(0, t)=-\frac{1}{\sqrt{3}} & \text { for all } t \in \mathbb{R} \\ \frac{-\Gamma_{x x}}{\left(1+\Gamma_{x}^{2}\right)^{3 / 2}}(0, t)=\lambda-\tilde{\varepsilon} & \text { for all } t \in \mathbb{R} \\ \Gamma\left(0, t_{0}\right)=\tilde{u}\left(0, t_{0}\right), & \\ \Gamma(x, t) \rightarrow-\infty & \text { as } x \nearrow \zeta \text { for every } t \in \mathbb{R}\end{cases}
$$

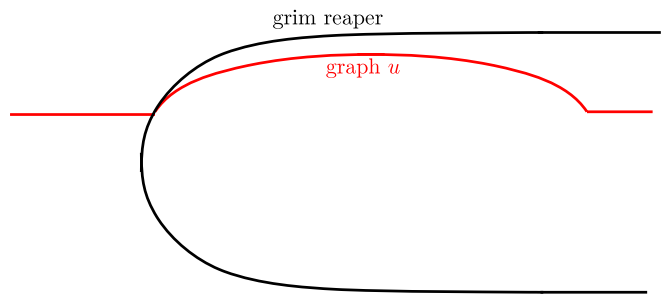

As the curvature of graph $\Gamma\left(\cdot, t_{0}\right)$ is bounded above by $\lambda-\tilde{\varepsilon}$ and the curvature of $M_{t_{0}}$ is bounded below by $\lambda-\tilde{\varepsilon}$ (with equality at the triple point), we see that $\delta<\zeta$ and

$$
\Gamma\left(x, t_{0}\right) \geq \tilde{u}\left(x, t_{0}\right) \text { for } x \in[0, \delta),
$$

with strict inequality near $x=\delta$. From the maximum principle it follows that

$$
\Gamma(x, t) \geq \tilde{u}(x, t) \text { for all }(x, t) \in[0, \delta) \times\left[t_{0}, \tau\right) .
$$

Moreover, our construction implies that the curvatures of graph $\tilde{u}\left(\cdot, t_{0}\right)$ and of graph $\Gamma\left(\cdot, t_{0}\right)$ coincide for $x=0$. Thus the evolution equation and the boundary condition imply that

$$
\dot{\Gamma}\left(0, t_{0}\right)=\dot{\tilde{u}}\left(0, t_{0}\right)=\frac{\tilde{u}_{x x}}{\left(1+\tilde{u}_{x}^{2}\right)^{3 / 2}} \sqrt{1+\tilde{u}_{x}^{2}}\left(0, t_{0}\right)=-\frac{2}{\sqrt{3}} \kappa\left(0, t_{0}\right) .
$$


Combining (3.4), (3.5) and (3.6) we conclude that $\ddot{\Gamma}\left(0, t_{0}\right) \geq \ddot{\tilde{u}}\left(0, t_{0}\right)$ and thus

$$
\dot{\kappa}\left(0, t_{0}\right)=-\frac{\sqrt{3}}{2} \ddot{\tilde{u}}\left(0, t_{0}\right) \geq-\frac{\sqrt{3}}{2} \ddot{\Gamma}\left(0, t_{0}\right) .
$$

Since graph $\Gamma(\cdot, t)$ is a translating solution we have $\ddot{\Gamma}=0$. This fact combined with the equation above implies $\dot{\kappa}\left(0, t_{0}\right) \geq 0$, which contradicts (3.2) and finishes the proof in the strictly convex case.

Now the general case follows by approximation. Indeed every convex initial condition may be approximated in $C^{2}$ by strictly convex networks. Standard results (see also [13] and Theorem 2.1) imply that solutions for this Neumann problem are in $C^{2 ; 1}$ and depend continuously on the initial data. Moreover, by our previous proof we have that the approximations stay strictly convex and $C^{2}$-close to our network for some positive time interval. The lemma follows by taking limits.

In what follows we consider the reduced network $G^{\prime}$ consisting of the network $G$ without the two open half-lines. We often identify $G^{\prime}$ with $F\left(G^{\prime}, t\right)$. The coming lemmata prove that along the flow $(F(\cdot, t))_{t \in[0, T)}$ (always restricted to $G^{\prime}$ ) the ratio of extrinsic and (modified) intrinsic distance defined in [12] is bounded from below.

Recall that the extrinsic distance $d_{\mathrm{ex}}$ is given by

$$
d_{\mathrm{ex}}: G^{\prime} \times G^{\prime} \times[0, T) \rightarrow \mathbb{R}, \quad d_{\mathrm{ex}}(p, q, t)=\|F(p, t)-F(q, t)\|_{2},
$$

where $\|\cdot\|_{2}$ denotes the Euclidean norm on $\mathbb{R}^{2}$. The intrinsic distance $d_{\text {in }}$ can be computed as

$$
d_{\text {in }}: G^{\prime} \times G^{\prime} \times[0, T) \rightarrow \mathbb{R}, \quad d_{\text {in }}(p, q, t)=\int_{p}^{q} d s_{t},
$$

where $s_{t}$ is the arc-length parameter at time $t$. Note that there are two locally minimizing paths from $p$ to $q$ in $F\left(G^{\prime}, t\right)$ of lengths smaller than the total length of $F\left(G^{\prime}, t\right)$. Let $d_{\text {in }}$ be the length of the shorter path $\gamma_{\text {in }}$, which is less than or equal to $L / 2$. Then the modified intrinsic distance is given by $\psi=\frac{L}{\pi} \sin \left(\frac{\pi d_{\text {in }}}{L}\right)$. Note that $\psi$ is smooth if $d_{\text {in }}>0$.

Proposition 3.4. Consider a family of convex symmetric networks solving (1.1) (or equivalently (2.1)). Suppose that at time $t_{0} \in[0, T)$ the quotient $\frac{d_{\mathrm{ex}}}{\psi}$ attains a spatial minimum at $(p, q)$ such that $p \neq q$ and neither $p$ nor $q$ is a triple point. Then

$$
\left.\frac{d}{d t} \frac{d_{\mathrm{ex}}}{\psi}(p, q, t)\right|_{t=t_{0}} \geq 0
$$

Proof. We reduce the calculation to the one done in 12 in the regular case. In order to do this we show that the terms obtained when computing $\frac{d}{d t} \frac{d_{\mathrm{ex}}}{\psi}$ are equal to the ones in [12] plus extra positive contributions coming from the triple points. Let us first compute $\frac{d}{d t} L=\dot{L}, \frac{d}{d t} d_{\text {in }}=\dot{d_{\text {in }}}$ and $\frac{d}{d t} \psi=\dot{\psi}$. Since

$$
L(t)=2 \int_{a(t)}^{b(t)} d s_{t}=2 \int_{a(t)}^{b(t)} \sqrt{1+u_{x}^{2}} d x
$$


we have

$$
\begin{aligned}
\frac{d}{d t} L(t) & =2 \int_{a(t)}^{b(t)} \frac{u_{x} \dot{u}_{x}}{\sqrt{1+u_{x}^{2}}} d x+2 \sqrt{1+u_{x}^{2}(b(t), t)} \cdot \dot{b}(t)-2 \sqrt{1+u_{x}^{2}(a(t), t)} \cdot \dot{a}(t) \\
& =2 \int_{a(t)}^{b(t)} \frac{u_{x}}{\sqrt{1+u_{x}^{2}}}\left(\frac{u_{x x}}{1+u_{x}^{2}}\right)_{x} d x+4 \dot{b}(t)-4 \dot{a}(t) .
\end{aligned}
$$

By differentiating $u(a(t), t)=0$ and $u(b(t), t)=0$ with respect to $t$ and using (2.1) we obtain $u_{x x}(a(t), t)=-4 \sqrt{3} \dot{a}(t)$ and $u_{x x}(b(t), t)=4 \sqrt{3} \dot{b}(t)$. Now integrating by parts it follows that

$$
\begin{aligned}
\frac{d}{d t} L(t) & =-2 \int_{a(t)}^{b(t)}\left(\frac{u_{x}}{\sqrt{1+u_{x}^{2}}}\right) \frac{u_{x x}}{1+u_{x}^{2}} d x+\dot{b}(t)-\dot{a}(t) \\
& =-2 \int_{a(t)}^{b(t)} \kappa^{2} \sqrt{1+u_{x}^{2}} d x+\dot{b}(t)-\dot{a}(t) \\
& =-2 \int_{(a(t), 0)}^{(b(t), 0)} \kappa^{2} d s_{t}+[\dot{b}(t)-\dot{a}(t)] .
\end{aligned}
$$

Here we used $\kappa^{2}=\frac{u_{x x}^{2}}{\left(1+u_{x}^{2}\right)^{3}}$. The first term appears in the computations in [12] while the second is a consequence of the triple points.

If neither $p$ nor $q$ is a triple point a similar calculation implies

$$
\frac{d}{d t} d_{\mathrm{in}}= \begin{cases}-\int_{p}^{q} \kappa^{2} d s_{t}-\dot{a} & \text { if }(a(t), 0) \in \gamma_{\mathrm{in}}, \\ -\int_{p}^{q} \kappa^{2} d s_{t}+\dot{b} & \text { if }(b(t), 0) \in \gamma_{\mathrm{in}} \\ -\int_{p}^{q} \kappa^{2} d s_{t} & \text { otherwise. }\end{cases}
$$

The chain rule and the computations above yield

$$
\begin{aligned}
\frac{d}{d t} \frac{d_{\mathrm{ex}}=}{\psi} \frac{\dot{d_{\mathrm{ex}}}}{\psi}-\frac{d_{\mathrm{ex}}}{\psi^{2}} \dot{\psi} \\
=\frac{\dot{d_{\mathrm{ex}}}}{\psi}-\frac{d_{\mathrm{ex}}}{\psi^{2}}\left(\frac{\dot{L}}{\pi} \sin \left(\frac{\pi d_{\mathrm{in}}}{L}\right)+\dot{d_{\mathrm{in}}} \cos \left(\frac{\pi d_{\mathrm{in}}}{L}\right)-\frac{\dot{L} d_{\mathrm{in}}}{L} \cos \left(\frac{\pi d_{\mathrm{in}}}{L}\right)\right) \\
=\frac{\dot{d_{\mathrm{ex}}}}{\psi}-\frac{d_{\mathrm{ex}}}{\psi^{2}}\left(-\frac{1}{\pi} \int_{G^{\prime}} \kappa^{2} d s_{t} \cdot \sin \left(\frac{\pi d_{\text {in }}}{L}\right)+\frac{d_{\text {in }}}{L} \int_{G^{\prime}} \kappa^{2} d s_{t} \cdot \cos \left(\frac{\pi d_{\mathrm{in}}}{L}\right)\right. \\
\left.\quad-\int_{p}^{q} \kappa^{2} d s_{t} \cdot \cos \left(\frac{\pi d_{\mathrm{in}}}{L}\right)\right)+B+C,
\end{aligned}
$$


where

$$
B=-\frac{d_{\text {ex }}}{\psi^{2}}\left(\frac{1}{\pi}(\dot{b}-\dot{a}) \sin \left(\frac{\pi d_{i n}}{L}\right)-\frac{d_{\text {in }}}{L}(\dot{b}-\dot{a}) \cos \left(\frac{\pi d_{\text {in }}}{L}\right)\right)
$$

and

$$
C= \begin{cases}\frac{d_{\mathrm{ex}}}{\psi^{2}} \dot{a} \cos \left(\frac{\pi d_{\mathrm{in}}}{L}\right) & \text { if }(a(t), 0) \in \gamma_{\mathrm{in}}, \\ -\frac{d_{\mathrm{ex}} \dot{b} \cos \left(\frac{\pi d_{\mathrm{in}}}{L}\right)}{\psi^{2}} & \text { if }(b(t), 0) \in \gamma_{\mathrm{in}}, \\ 0 & \text { otherwise. }\end{cases}
$$

Notice that besides $B$ and $C$ all the terms in the above computation appear as well in the regular case. Therefore, in order to conclude the result it is enough to show that $B, C \geq 0$. Because the lens is convex we have $\dot{a} \geq 0$ and $\dot{b} \leq 0$. Furthermore $0 \leq d_{\text {in }} \leq L / 2$, which implies $C \geq 0$. Similarly, recalling that for $y \geq 0$ it holds that $\tan y \geq y$, we get

$$
B \cdot \frac{\psi^{2}}{d_{e x}}=\frac{1}{\pi}(\dot{a}-\dot{b})\left(\sin \left(\frac{d_{\mathrm{in}} \pi}{L}\right)-\frac{d_{\mathrm{in}} \pi}{L} \cos \left(\frac{d_{\mathrm{in}} \pi}{L}\right)\right) \geq 0 .
$$

This result together with the following lemma gives us the desired bound from below on the ratio of extrinsic and (modified) intrinsic distance.

Lemma 3.5. Consider a family of convex symmetric networks solving (1.1) (or equivalently (2.1)). If at a time $t \in[0, T)$ the quotient $d_{\mathrm{ex}} / \psi$ attains a spatial minimum smaller than $\sqrt{3} / 2$ at $(p, q)$, then neither $p$ nor $q$ can be a triple point. Thus we have

$$
\inf _{p \neq q} \frac{d_{\mathrm{ex}}(p, q, t)}{\psi(p, q, t)} \geq \min \left\{\inf _{p \neq q} \frac{d_{\mathrm{ex}}(p, q, 0)}{\psi(p, q, 0)}, \frac{\sqrt{3}}{2}\right\}
$$

for all $t \in[0, T)$.

Proof. We fix $t$. Near the triple points the network is approximately straight. Hence, geometric considerations show that

$$
\liminf _{p, q} \frac{d_{\mathrm{ex}}(p, q, t)}{\psi(p, q, t)}=\frac{\sqrt{3}}{2}
$$

if $p$ and $q$ approach a triple point from two different sides. If they approach another point or the triple point from the same side, the limit is 1 .

If $p$ and $q$ are both triple points it is easy to see that we can decrease $d_{\mathrm{ex}} / \psi$ by moving $p$ and $q$ clockwise (or counterclockwise) along the lens maintaining the intrinsic distance $d_{\text {in }}(p, q, t)$ (and thus maintaining the value of $\psi(p, q, t)$ ). So we restrict ourselves to the case that $p$ is the left triple point and $q$ lies on the upper part of the lens. The idea here is again to move $p$ and $q$ either clockwise or counterclockwise along the lens maintaining $d_{\text {in }}(p, q, t)$ and decreasing $d_{\text {ex }}(p, q, t)$.

Let $\vec{n}^{+}$and $\vec{n}^{-}$denote the two unit tangent vectors at the left triple point $p$, pointing to the right and into the upper half-plane and into the lower half-plane, respectively. Let $\vec{\tau}$ denote the unit tangent vector at $q$ pointing towards $p$. Since the lens is convex, we see that if $\vec{\tau}$ is not parallel to $\vec{n}^{-}$, we decrease $d_{\text {ex }}(p, q, t)$ by moving both points counter clockwise as $\left\langle\vec{n}^{-}, \vec{\tau}\right\rangle<0$. If $\vec{\tau}$ is parallel to $\vec{n}^{-}$we can decrease $d_{\mathrm{ex}}(p, q, t)$ by moving both points clockwise. 
Since $d_{\text {in }} \leq L / 2$ we have $\psi \geq \frac{2}{\pi} d_{\text {in }}$. This implies

Corollary 3.6 (Bounds on intrinsic and extrinsic distances). Consider a family of convex symmetric networks solving (1.1). We have

$$
\frac{d_{\text {ex }}(p, q, t)}{d_{\text {in }}(p, q, t)} \geq \frac{2}{\pi} \min \left\{\inf _{r \neq s} \frac{d_{\text {ex }}(r, s, 0)}{\psi(r, s, 0)}, \frac{\sqrt{3}}{2}\right\}
$$

for all $p, q \in G^{\prime}, p \neq q$ and $t \in[0, T)$.

We finish this section by showing:

Proposition 3.7 (Curvature bounds). Let $\left(M_{t}\right)_{t \in[0, T)}$ be a family of convex symmetric networks solving (1.1) (or, equivalently, (2.1)). Then the curvature of $M_{t}$ is bounded everywhere in terms of a positive lower bound for $T-t, \sup \kappa(\cdot, 0)$, and a bound on the quotient of intrinsic and extrinsic distances as obtained in Corollary 3.6.

Theorem 1.1 is a direct consequence of the curvature bounds. That is, we may choose $T$ in (2.1) such that $\left|\Omega_{t}\right| \searrow 0$ as $t \nearrow T$; i.e., a solution exists until the enclosed volume goes to zero.

We would like to remark that from the proof of Proposition 3.7 we obtain, as long as $\left|\Omega_{t}\right|$ is uniformly bounded below, uniform curvature estimates for the sequence of rescaled solutions considered in Section 4 .

Proof of Proposition 3.7. We organize the coming proof as follows. We start by controlling the geometry of the network, using the previous bounds on $d_{\mathrm{ex}} / d_{\mathrm{in}}$. More precisely, we conclude that sup $|u(\cdot, t)|, \sqrt{\left|\Omega_{t}\right|}$ and $|a(t)-b(t)|$ are comparable. This allows us to use interior estimates to bound the curvature at a given distance away from the triple points. We conclude by controlling the curvature up to the triple points via the maximum principle.

Geometric control. Assume that $0<\frac{1}{c} \leq T-t \leq c$. From Lemma 3.2 we get similar bounds for $\left|\Omega_{t}\right|$. As usual, in view of the short-time existence, we may assume that we have a smooth solution up to a certain time, up to which we want to prove a priori estimates.

We claim that there exists a constant $c$ depending only on $d_{\text {in }} / d_{\text {ex }}$, such that

$$
\frac{1}{c} \cdot|a(t)-b(t)| \leq \sup _{x \in(a(t), b(t))}|u(x, t)| \leq c \cdot|a(t)-b(t)|
$$

and

$$
\frac{1}{c} \cdot\left|\Omega_{t}\right| \leq|a(t)-b(t)|^{2} \leq c \cdot\left|\Omega_{t}\right| \cdot
$$

These can be seen as follows: Convexity implies the gradient estimate $-\sqrt{3} \leq$ $u_{x}(x, t) \leq \sqrt{3}$ for all $x, t$. From this follows the upper bound in the first inequality. We prove the lower bound by contradiction. If there is a sequence $t_{i}$ such that $\frac{\sup \left|u\left(\cdot, t_{i}\right)\right|}{a\left(t_{i}\right)-b\left(t_{i}\right)} \rightarrow 0$, the lens-shaped domain would be very thin, contradicting the upper bound on $d_{\text {in }} / d_{\text {ex }}$.

Using convexity again, we see that $\left|\Omega_{t}\right|$ is comparable to

$$
|a(t)-b(t)| \cdot \sup _{x \in(a(t), b(t))}|u(x, t)|
$$

which implies the second inequality. 
Curvature bounds. The short-time existence proof implies the claimed estimates as long as $t$ is small in terms of $\sup |u(\cdot, 0)|, d_{\text {in }} / d_{\text {ex }}$ and $\sup \kappa(\cdot, 0)$.

For $t \geq \frac{1}{c}>0$, we will use the following interior estimate, obtained from [4, 5, 6, 7]: Let $u:(-2 r, 2 r) \times\left(0,4 r^{2}\right) \rightarrow \mathbb{R}$ for some $r>0$ be a solution to $\dot{u}=\frac{u_{x x}}{1+u_{x}^{2}}$. Then there exist constants $c_{k}$ that depend on $k, r$ and $\|u\|_{C^{0}\left((-2 r, 2 r) \times\left(0,4 r^{2}\right)\right)}$ such that

$$
\sup _{(x, t) \in(-r, r) \times\left(3 r^{2}, 4 r^{2}\right)}\left|\frac{\partial^{k} u}{\partial x^{k}}\right|(x, t) \leq c_{k} .
$$

We would like to remark that in our situation even the weaker estimates in 7 would suffice.

Fix $0<\varepsilon<\frac{1}{4}$ and define

$$
M:=\inf _{t} \sup _{x}|u(x, t)|
$$

Assume that we are in the set where $u \geq \varepsilon \cdot M$. Since $\left|u_{x}\right| \leq \sqrt{3}$, we can find $\delta>0$ such that this set is contained in $[a(t)+\delta, b(t)-\delta] \times \mathbb{R}$. Note that $\dot{a} \geq 0$ and $\dot{b} \leq 0$. Thus we can apply the interior estimates (3.7) and get the claimed a priori estimates in the set where $u \geq 2 \varepsilon \cdot M$.

Suppose now that we are in the domain where $u \leq(1-\varepsilon) \cdot M$. In this set, convexity implies that $\left|u_{x}\right|$ is bounded below by $\frac{\varepsilon \cdot M}{|a(t)-b(t)|}$. Thus, rotating the coordinate system by $\pi / 2$, graph $\left.u\right|_{(a(t), b(t))}$ is represented in each connected component of this domain as a graph with bounded gradient.

Consider graphs as in (3.3). Due to the curvature estimates away from the triple point obtained above, we may assume that we have $C^{2}$ bounds if $x$ is bounded away from zero, i.e. especially for some fixed positive $x$. Consider $V:=-\dot{u}=-\frac{u_{x x}}{1+u_{x}^{2}}$ corresponding to $\kappa v$ in standard covariant notation. We have the evolution equation

$$
\frac{d}{d t} V=\frac{1}{1+u_{x}^{2}} V_{x x}-\frac{2 u_{x} u_{x x}}{\left(1+u_{x}^{2}\right)^{2}} V_{x} .
$$

Differentiating the boundary condition yields $V_{x}=0$ at the triple point. Now the claim follows from the maximum principle.

\section{Proof of Theorem 1.3}

In this section we combine the bounds proved in the previous section and Huisken's monotonicity formula to show that a given solution converges under rescaling to the unique symmetric self-similarly shrinking solution described in Theorem 1.2

Let us consider a network $M_{t}$ for $t \in[0, T)$ that contracts to a point $x_{0}$ on the $x^{1}$-axis as $t \nearrow T$. Consider a sequence of positive real numbers $\lambda_{i} \nearrow \infty$ satisfying $\lambda_{i}^{2} T>1$. We rescale the network under a sequence of parabolic dilations given by

$$
M_{\tau}^{i}:=\lambda_{i}\left(M_{\lambda_{i}^{-2} \tau+T}-x_{0}\right) .
$$

It is easy to see that for each $i$ the rescaled network $M_{\tau}^{i}$ satisfies the mean curvature flow equation in the time interval $\left[-\lambda_{i}^{2} T, 0\right)$. Notice that this parabolic rescaling also preserves the symmetry, the gradient estimates and the $120^{\circ}$ condition. In 
particular, the rescaled networks are solutions described by a graph, and the ratio estimate from Corollary 3.6.

$$
d_{\text {in }} \leq C d_{\mathrm{ex}}
$$

is preserved.

From the geometric estimates in the proof of Proposition 3.7, we deduce

Corollary 4.1. The networks $M_{\tau}^{i}$ are uniformly bounded in $C^{\infty}$ independently of $i$ on any compact time subinterval of $(-1,0)$.

Proof of Theorem 1.3. Note that since the networks $M_{t}$ (respectively $M_{\tau}^{i}$ ) satisfy the mean curvature flow equation they can be seen as Brakke flows with equality (see [13]).

Huisken's monotonicity formula $([11,13])$ implies that the Gaussian density ratio given by

$$
\Theta\left(M_{t}, x_{0}, T\right)=\int_{M_{t}} \frac{1}{(4 \pi(T-t))^{\frac{1}{2}}} \exp \left(-\frac{\left|x-x_{0}\right|^{2}}{4(T-t)}\right) d \mu \quad \text { for } t<T
$$

is monotonically decreasing in time. Therefore, the limit

$$
\Theta\left(x_{0}, T\right):=\lim _{t \nearrow T} \Theta\left(M_{t}, x_{0}, t\right)
$$

exists and is finite. This limit is known as the Gaussian density and it satisfies

$$
\Theta\left(M_{t}, x_{0}, t\right)-\Theta\left(x_{0}, T\right)=\int_{t}^{T} \int_{M_{t}} \rho_{x_{0}, T}(x, t)\left|\kappa-\frac{\left\langle x-x_{0}, \nu\right\rangle}{2(T-t)}\right|^{2} d \mu_{t}(x) d t
$$

where $\rho_{x_{0}, T}(x, t):=(4 \pi(T-t))^{-1 / 2} \exp \left(-\frac{\left|x-x_{0}\right|^{2}}{4(T-t)}\right)$.

Changing variables according to the parabolic rescaling described by (4.1) we obtain

$$
\Theta\left(M_{t}, x_{0}, t\right)-\Theta\left(x_{0}, T\right)=\int_{\lambda_{i}^{2}(t-T)}^{0} \int_{M_{\tau}^{i}} \rho_{0,0}(y, \tau)\left|\kappa+\frac{\langle y, \nu\rangle}{2 \tau}\right|^{2} d \mu_{\tau}(y) d \tau .
$$

Now for each $\lambda_{i}$ we look at the flow $M_{\tau}^{i}$ at time $\tau=-1 / 2$. This defines a sequence $t_{i} \rightarrow T$ satisfying $-\lambda_{i}^{-2} \frac{1}{2}+T=t_{i}$. Using Corollary 4.1 we can extract a subsequence, which we again call $M_{\tau}^{i}$, that converges to a limit network $M_{\tau}^{\prime}$ and satisfies all the estimates above and, as before, flows by mean curvature.

Moreover, (4.2) implies

$$
\int_{-1}^{0} \int_{M_{\tau}^{i}} \rho_{0,0}(y, \tau)\left|\kappa+\frac{\langle y, \nu\rangle}{2 \tau}\right|^{2} d \mu_{\tau}(y) d \tau \rightarrow 0 .
$$

Thus $M_{-\frac{1}{2}}^{\prime}$ satisfies

$$
\kappa=\langle x, \nu\rangle
$$

and $\left(M_{\tau}^{\prime}\right)_{\tau<0}$ is a self-similarly shrinking solution which by the results of Section 5 and the symmetry assumption is unique.

Since the limiting flow does not depend on the sequence $\left(\lambda_{i}\right)_{i}$ chosen, we obtain the stated result by choosing an appropriate sequence $\left(\lambda_{i}\right)_{i}$. 


\section{Existence AND UNiqUENESS OF SELF-Similar LENSES}

In this section we prove Theorem [1.2. We divide the proof into two subsections: existence of self-similar shrinking lenses and uniqueness in this class.

5.1. Existence. We investigate the existence of a homothetically shrinking solution $u(x, t)$ of (2.1). That is, we study the existence of a profile $u_{0}$, a scaling function $\lambda=\lambda(t)$ and an interval $I$ such that

$$
\{(x, u(x, t)): x \in \lambda(t) I\}=\lambda(t) \cdot\left\{\left(y, u_{0}(y)\right): y \in I\right\} .
$$

By letting $x=\lambda(t) y$ we obtain

$$
u(x, t)=\lambda(t) \cdot u_{0}\left(\frac{x}{\lambda(t)}\right) .
$$

From the evolution equation (2.1), we get

$$
\frac{u_{0}^{\prime \prime}}{1+\left(u_{0}^{\prime}\right)^{2}}=\lambda(t) \dot{\lambda}(t)\left(-\frac{x}{\lambda(t)} u_{0}^{\prime}+u_{0}\right)=\lambda(t) \dot{\lambda}(t)\left(-y u_{0}^{\prime}+u_{0}\right) .
$$

This implies $\frac{d}{d t}\left(\frac{1}{2} \lambda^{2}\right)=$ const. Since $u(x, t)$ is a shrinking solution, it necessarily follows that $\dot{\lambda}(t)<0, \lambda(t)>0$. By assuming that $\lambda(-1 / 2)=1$ and fixing the blowup time to be $T=0$ we obtain $\lambda(t)=\sqrt{-2 t}$. For simplicity, in the remainder of this section we relabel $u:=u_{0}$ and $x:=y$. Then, we obtain the following equation that determines the self-similarly shrinking solution:

$$
u^{\prime \prime}=\left(1+u^{\prime 2}\right)\left(x u^{\prime}-u\right) \text {. }
$$

(Note that this equation is equivalent to $\kappa=\langle(x, u), \nu\rangle$.) Thus, the existence statement of Theorem 1.2 can be reduced to the following proposition.

Proposition 5.1. There exists a solution $u$ to (5.2) that via (5.1) induces a homothetically shrinking solution to (2.1) for $-\infty<t<0$. Moreover, this solution is symmetric with respect to the $x^{2}$-axis.

Proof. Given $0<h<1$ let us consider the initial value problem

$$
\left\{\begin{array}{l}
u_{x x}=\left(1+u_{x}^{2}\right)\left(u_{x} x-u\right), \quad 0 \leq x \leq x_{\max } \\
u(0)=h \\
u_{x}(0)=0
\end{array}\right.
$$

We denote the solution to this equation by $u^{h}$. In order to prove the proposition we show that there is an $0<H<1$ and a point $x_{0}^{H}$ such that $u^{H}\left(x_{0}^{H}\right)=0, u^{H}(x)>0$ for $x \in\left[0, x_{0}^{H}\right)$ and $u_{x}^{H}\left(x_{0}^{H}\right)=-\sqrt{3}$. Then $u^{H}$ induces a homothetically shrinking solution symmetric with respect to the $x^{2}$-axis.

In order to find $H$, consider

$$
\begin{gathered}
\mathcal{S}:=\left\{h \in(0,1): \exists x_{0}^{h} \in(0, \sqrt{2}] \text { such that } u^{h}(x)>0 \text { for } x \in\left[0, x_{0}^{h}\right),\right. \\
\left.u^{h}\left(x_{0}^{h}\right)=0 \text { and }\left|u_{x}^{h}\right|<\sqrt{3} \text { on }\left[0, x_{0}^{h}\right]\right\}
\end{gathered}
$$

and define $H:=\sup \mathcal{S}$. We will show that this $H$ satisfies the properties required above.

In order to prove this we show that $\mathcal{S}$ is not empty and open. Moreover, we prove that $H<1$. This implies that for $h=H$ necessarily one of the conditions 
that define $\mathcal{S}$ has to be violated. We will conclude the result from the existence of $x_{0}^{H}$ such that $u^{H}(x)>0$ for $x \in\left[0, x_{0}^{H}\right), u^{H}\left(x_{0}^{H}\right)=0$ and $\left|u_{x}^{H}\right|\left(x_{0}^{H}\right) \geq \sqrt{3}$.

Lines through the origin are solutions to (5.2). Hence uniqueness of solutions to ordinary differential equations implies that our solutions stay concave; i.e. $u_{x x}^{h}<0$. Since $\left(x u_{x}^{h}-u^{h}\right)_{x}=x u_{x x}^{h}<0$ we get

$$
u_{x x}^{h}=\left(1+\left(u_{x}^{h}\right)^{2}\right)\left(x u_{x}^{h}-u^{h}\right) \leq x u_{x}^{h}-u^{h} \leq-u^{h}(0)=-h,
$$

and therefore $u^{h}(x) \leq h-\frac{1}{2} h x^{2}$. In particular, if the gradient of $u^{h}$ remains bounded, there is an $0 \leq x_{0}^{h} \leq \sqrt{2}$ such that $u^{h}(x)>0$ for $x \leq x_{0}^{h}$ and $u^{h}\left(x_{0}^{h}\right)=0$.

It is easy to check that $u^{0} \equiv 0$. Continuous dependence on the initial data implies that for $|h| \ll 1$ sufficiently small, $\left|u_{x}^{h}\right|<\frac{\sqrt{3}}{2}$ holds in $[0, \sqrt{2}]$. Thus we can fix $\varepsilon>0$ such that there exists $x_{0}^{\varepsilon}>0$ satisfying $u^{\varepsilon}\left(x_{0}^{\varepsilon}\right)=0$, and $u^{\varepsilon}>0, u_{x}^{\varepsilon}>-\sqrt{3}$ in $\left[0, x_{0}^{\varepsilon}\right]$. In particular, $\mathcal{S} \neq \emptyset$.

On the other hand, it is also easy to check that graph $u^{1}$ lies on the unit circle. Hence, there is $x_{\sqrt{3}}^{1}$ such that $0<x_{\sqrt{3}}^{1}<1, u_{x}^{1}\left(x_{\sqrt{3}}^{1}\right)=-\sqrt{3}$ and $u^{1}>0$ in $\left[0, x_{\sqrt{3}}^{1}\right]$. Denote by $x_{\sqrt{3}}^{h}$ the value where

$$
u_{x}^{h}\left(x_{\sqrt{3}}^{h}\right)=-\sqrt{3} .
$$

As solutions are strictly concave, $x_{\sqrt{3}}^{h}$ depends smoothly on $h$ near $h=1$. Since $u^{1}$ does not extend up to $x=1$ and $u^{1}(x)>0$ for $x \in[0,1)$, it holds that $H<1$.

Now, in order to show that $\mathcal{S}$ is open we note that $u^{h}$ is strictly decreasing in $x$ for $x>0$ and $h>0$, and the value $x_{0}^{h}>0$ (where the solution $u^{h}$ becomes zero) depends smoothly on $h$ if $u_{x}^{h}\left(x_{0}^{h}\right)>-\infty$. Similarly, since for $h \in \mathcal{S}$ the solution $u_{x}^{h}$ does not blow up in $\left[0, x_{0}^{h}\right], u_{x}^{h}$ depends smoothly on $h$ as well. This implies $\mathcal{S}$ is open.

Consider $h_{i} \in \mathcal{S}$ such that $h_{i} \rightarrow H$. The previous claims imply that $\lim x_{0}^{h_{i}}=$ $x_{0}^{H} \leq \sqrt{2}$ and $u_{x}^{H}\left(x_{0}^{H}\right) \geq-\sqrt{3}$, which finishes the proof.

5.2. Uniqueness of self-similar lenses. The main result of this section is:

Lemma 5.2. The solution obtained in Proposition 5.1 is unique in the class of lens-shaped networks. In particular, the solution is symmetric with respect to the $x^{2}$-axis, that is, $u_{x}(0)=0$.

We divide the estimates in this section into two types: geometric estimates that provide information for small heights $h$ (for the definition of height, see Section 5.2.1), and integral estimates inspired by [2].

5.2.1. Geometric estimates. Let us recall some properties of homothetically shrinking solutions to curve shortening flow; see [1, 2] for details. We normalize these solutions as in Section 5.1. Such curves wind around the origin and may or may not close up after finitely many turns. In this paragraph, we will not consider the trivial case when the curve is the unit circle. If we follow such a curve, we will encounter minima and maxima of the distance to the origin. The distance of the minima from the origin is less than one, whereas the distance of the maxima from the origin is greater than one. Between a minimum and an adjacent maximum, the distance to the origin is strictly monotone. The angle between adjacent minima and maxima is bounded from below by $\frac{\pi}{2}$ and from above by $\frac{\pi}{\sqrt{2}}$. Moreover, the 


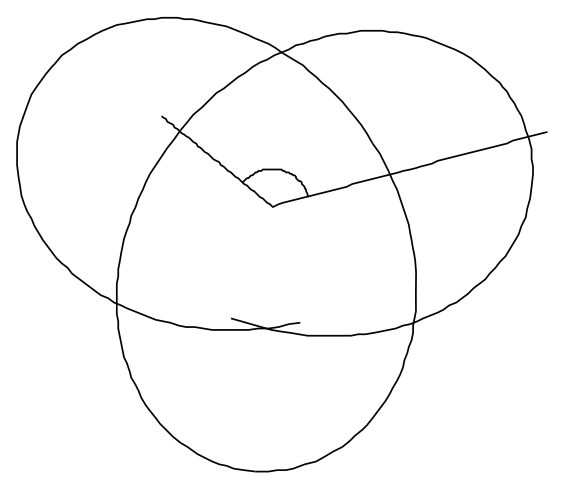

FiguRE 3. Extrema on self-similar solutions

part of the curve between such extremal points is the building block for homothetically shrinking solutions: Reflecting it at a line through the origin and one of its endpoints and repeating this process with the new endpoints, we obtain the entire curve. See Figure 3.

Consider graph $u$ in $\left\{x^{2}>0\right\}$, where $u$ solves (5.2) and $u_{x}= \pm \sqrt{3}$ at the respective endpoints. Then there exists a unique point on graph $u$ minimizing the distance to the origin: Near the endpoints, the boundary condition ensures that the distance is strictly decreasing on the left-hand side and strictly increasing at the right-hand side. Hence there exists at least one minimum. The bounds on the angle between adjacent minima and maxima ensure that there exists at most one extremal point (besides the endpoints) of the distance function to the origin on graph $u$.

Now let $P$ be the point on graph $u$ minimizing the distance to the origin. In polar coordinates we describe this point by $r=h$ and $\varphi=\beta$. Without loss of generality we assume that this point lies on the right upper quadrant. By defining $b$ as the distance from the origin to the point where $u$ intersects the $x^{1}$-axis we have the picture in Figure 4

In particular, lenses that are symmetric with respect to the $x^{2}$-axis satisfy $\beta=\frac{\pi}{2}$.

We use the following energy, which is a first integral for the equation of a selfsimilarly shrinking solution:

$$
E^{u}:=\langle F, \nu\rangle e^{-\frac{1}{2}|F|^{2}} \equiv \frac{u-x u_{x}}{\sqrt{1+u_{x}^{2}}} e^{-\frac{1}{2}\left(x^{2}+u^{2}\right)} .
$$

This energy was already applied by Uwe Abresch and Joel Langer [1 to classify all closed and immersed self-similarly shrinking curves. Later on, Ben Andrews 2 applied a modified energy to classify such solutions when the normal speed of the evolution is a positive power of the curvature.

Lemma 5.3. The energy $E^{u}$ is constant in space along self-similar solutions $F$ of (1.1) with $T-t=\frac{1}{2}$ or, equivalently, independent of $x$ for solutions u to (5.2). 


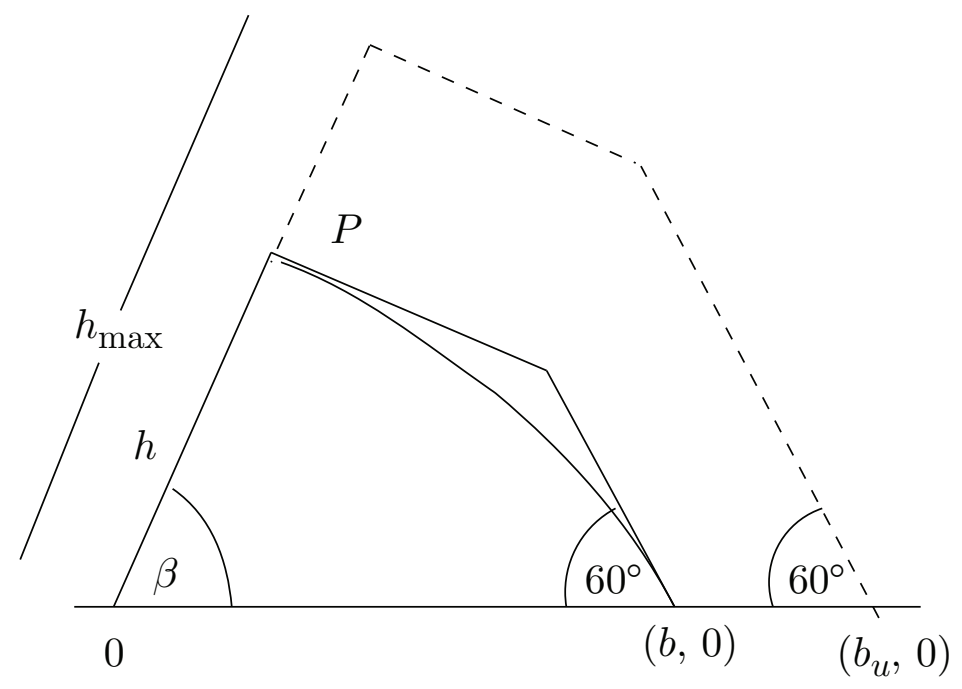

Figure 4. Circumscribed quadrilateral

Proof. Direct computations using (5.2) give

$$
\begin{aligned}
E_{x}^{u} \sqrt{1+u_{x}^{2}} e^{\frac{1}{2}\left(x^{2}+u^{2}\right)=} & -x u_{x x}\left(1+u_{x}^{2}\right)-u_{x} u_{x x}\left(u-x u_{x}\right) \\
& -\left(u-x u_{x}\right)\left(1+u_{x}^{2}\right)\left(x+u u_{x}\right) \\
= & u_{x x}\left[-x\left(1+u_{x}^{2}\right)-u_{x}\left(u-x u_{x}\right)+\left(x+u u_{x}\right)\right]=0 .
\end{aligned}
$$

In what follows, when we use explicit numbers, the corresponding estimates can be verified easily by hand.

In what remains of this subsection we will limit ourselves to the case $h \leq 0.5869 \equiv$ $h_{\text {max }}$.

Moreover, suppose that $(b, 0)$ corresponds to the intersection point of the selfsimilar curve and the positive $x^{1}$-axis. Then the following lemma holds:

Lemma 5.4. If $h \leq h_{\max }$ and a solution intersects the $x^{1}$-axis at an angle of $60^{\circ}$, then $b \leq 0.7645 \equiv b_{u}$ or $b \geq 1.2568 \equiv b_{l}$.

Proof. By Lemma 5.3, applied to $P$ and $(b, 0)$, we have that

$$
h e^{-\frac{h^{2}}{2}}=\sin \frac{\pi}{3} \cdot b e^{-\frac{b^{2}}{2}} \text {. }
$$

Since $0 \leq h \leq h_{\max }$ and $h \mapsto h \exp \left(-h^{2} / 2\right)$ is increasing on $[0,1]$, we have

$$
\sin \frac{\pi}{3} \cdot b e^{-\frac{b^{2}}{2}} \leq 0.5869 e^{-\frac{0.5869^{2}}{2}} \leq 0.49405 \text {. }
$$

Moreover,

$$
\begin{aligned}
& \sin \frac{\pi}{3} \cdot b e^{-\frac{b^{2}}{2}} \geq \sin \frac{\pi}{3} \cdot 0.7645 e^{-\frac{0.7645^{2}}{2}} \geq 0.49430 \text { for } 1 \geq b \geq 0.7645 \\
& \sin \frac{\pi}{3} \cdot b e^{-\frac{b^{2}}{2}} \geq \sin \frac{\pi}{3} \cdot 1.2568 e^{-\frac{1.2568^{2}}{2}} \geq 0.49408 \text { for } 1 \leq b \leq 1.2568
\end{aligned}
$$


The following proposition states that if $h \leq h_{\max }$, there are no solutions to (5.2) such that graph $u$ intersects the $x^{1}$-axis on both sides at an angle of $60^{\circ}$.

Proposition 5.5. Assume that $0<h \leq h_{\max }$. Then there is no solution $u$ to (5.2) such that $u_{x}(b)=-\sqrt{3}$ and $u_{x}(a)=\sqrt{3}$, where $a$ is defined as $b$, but for the negative $x^{1}$-axis.

The proof will be done by contradiction and it is divided into two cases: $b \geq b_{l}$ and $b \leq b_{u}$. In order to obtain a contradiction in the first case we will build a barrier function, for which we will prove that it intersects the $x^{1}$-axis at $x^{1}<b_{l}$. For the latter case we will consider the polygon depicted by the dotted lines in Figure 4. This polygon has fixed sides $h_{\max }$ and $b_{u}$, but changes with the angle $\beta$. Moreover, if $b \leq b_{u}$, this polygon always contains the part of the solution shown in Figure 4. We obtain a contradiction by showing that the area of the polygon (denoted by $A_{\square}$ ) is always smaller than the area enclosed by the solution. In order to show this we will need:

Lemma 5.6. Let $A_{\square}$ be the area of the polygon with sides of length $h_{\max }$ and $b_{u}$, respectively, as shown in Figure 4. Then we have

$$
\frac{d A_{\square}}{d \beta}<\frac{1}{2} .
$$

Proof. Observe that $\frac{d A_{\square}}{d \beta}=\frac{1}{2} l^{2}$, where $l$ denotes the length of the diagonal of the quadrangle that starts at the origin. This can be seen by bisecting the quadrangle along that diagonal and then regarding the variation of area as $\beta$ increases as the extra triangle that appears next to this bisecting line.

We conclude by noting that $l^{2}$ is estimated from above by $h_{\max }^{2}+b_{u}^{2}<1$.

Now we proceed with the proof of the proposition.

Proof of Proposition 5.5. We first want to remark that results in [1, 2] show that between points of minimum distance and maximum distance to the origin there is at least an angle of $\frac{\pi}{2}$. As $h \leq 1$ and $\left|u_{x}\right| \leq \sqrt{3}$, above the $x^{1}$-axis the solutions stay graphical. Now we proceed with the two cases described above.

Consider $b>b_{l}$. We first consider the "symmetric case", i.e. the case $u_{x}(0)=0$. Assume that $x>0$. We know that $u_{x}<0$ and $u_{x x}<0$. Moreover, direct calculations and equation (5.2) imply that $u_{x x x}<0$ and $u_{x x x x}<0$. By defining $f(x):=x u_{x}-u$ we obtain for $k \in \mathbb{N}$,

$$
\frac{d^{k} f}{d x^{k}}(x)=(k-1) \frac{d^{k} u}{d x^{k}}(x)+x \frac{d^{k+1} u}{d x^{k+1}}(x) .
$$

Thus $f_{x}(x)=x u_{x x}<0, f_{x x}<0$, and $f_{x x x}<0$. Hence

$$
\begin{gathered}
\left(x u_{x}-u\right)(x)=f(x) \leq f(0)+f_{x}(0) \cdot x+\frac{1}{2} f_{x x}(0) \cdot x^{2}<-h-\frac{1}{2} h x^{2}, \\
u_{x x}(x)=\left(1+u_{x}^{2}\right)\left(x u_{x}-u\right)<-h\left(1+u_{x}^{2}\right)\left(1+\frac{1}{2} x^{2}\right) .
\end{gathered}
$$

As $u_{x}^{2} \geq 0$, we get $u_{x}(x)<-h x-\frac{1}{6} h x^{3}$ and $u(x)<h-\frac{1}{2} h x^{2}-\frac{1}{24} h x^{4}$. We see that $u(x)<0$ for $x \geq \sqrt{-6+2 \sqrt{15}}$. In particular, $0<b<\sqrt{-6+2 \sqrt{15}} \equiv B_{1}$. Using $x=0$ and $x=b$, we get according to Lemma 5.3 .

$$
E^{u}=h e^{-\frac{1}{2} h^{2}}=\sin \frac{\pi}{3} \cdot b \cdot e^{-\frac{1}{2} b^{2}} .
$$


As $x \mapsto x e^{-\frac{1}{2} x^{2}}$ is monotonically increasing for $0 \leq x \leq 1$ and monotonically decreasing for $1 \leq x$, we get for $h \leq H_{1} \leq 1$,

$$
H_{1} e^{-\frac{1}{2} H_{1}^{2}} \geq E^{u}>\sin \frac{\pi}{3} \cdot B_{1} e^{-\frac{1}{2} B_{1}^{2}} .
$$

A direct computation shows that this inequality is violated if $0<h \leq H_{1}:=0.5587$.

We assume now that $H_{1} \leq h \leq h_{\max }$. Then we have $u_{x}^{2} \geq h^{2}\left(x+\frac{1}{6} x^{3}\right)^{2}$. Thus,

$$
\begin{aligned}
u_{x x}(x) & \leq-h\left(1+h^{2}\left(x+\frac{1}{6} x^{3}\right)^{2}\right)\left(1+\frac{1}{2} x^{2}\right) \\
& =-h\left(1+\left(\frac{1}{2}+h^{2}\right) x^{2}+\frac{5}{6} h^{2} x^{4}+\frac{7}{36} h^{2} x^{6}+\frac{1}{72} h^{2} x^{8}\right), \\
u_{x}(x) & \leq-h\left(x+\frac{1}{3}\left(\frac{1}{2}+h^{2}\right) x^{3}+\frac{1}{6} h^{2} x^{5}+\frac{1}{36} h^{2} x^{7}+\frac{1}{648} h^{2} x^{9}\right), \\
u(x) & \leq h-h\left(\frac{1}{2} x^{2}+\frac{1}{12}\left(\frac{1}{2}+h^{2}\right) x^{4}+\frac{1}{36} h^{2} x^{6}+\frac{1}{288} h^{2} x^{8}+\frac{1}{6480} h^{2} x^{10}\right) .
\end{aligned}
$$

We obtain $b<1.2568$. Arguing as above, this yields $h>h_{\max }$, which contradicts the assumptions of the lemma.

Now we consider the case $u_{x}(0) \neq 0$. We may assume that $u_{x}(0)<0$. We start by rotating the coordinates such that the new $x^{2}$-axis agrees with the old line of slope $\tan \beta$. Using the first barrier from the argument in the symmetric case we see that we get a contradiction if the $x^{1}$-coordinate of the intersection of graph $u$ and the new $x^{1}$-axis is greater than $B_{1}$. The results in 2 imply that the distance to the origin is increasing up to the point where the maximum is attained. Thus we also get a contradiction if the distance to the origin at the intersection with the old $x^{1}$-axis is greater than $B_{1}$. Since we have assumed that our solution intersects this old $x^{1}$-axis at an angle of $60^{\circ}$, we can exclude initial heights $h$ with $0<h \leq H_{1}$ as before. Repeating this argument with the second barrier from the symmetric case yields the stated claim.

Consider $b<b_{u}$. As mentioned above, in this situation the strategy of the proof is to compute and compare the difference between the areas enclosed by the quadrilateral shown in Figure 4 and by the solution. We will observe that for $0<h \leq h_{\max }$, this difference is always negative, contradicting the definition of the polygon.

From [2, see also the beginning of Section 5.2.1] we obtain that the angle between a minimum and a maximum of $|F|=\sqrt{x^{2}+u^{2}}$ is at most $\frac{\pi}{\sqrt{2}}$. Moreover, this angle is at least $\frac{\pi}{2}$. Thus the boundary conditions imply that there is precisely one minimum of $|F|$ above the $x^{1}$-axis. Hence $\beta>\pi-\frac{\pi}{\sqrt{2}}$; for otherwise, $|F|$ would have a local maximum between $P$ and the negative $x^{1}$-axis.

The area $A$ of the domain bounded by the self-similarly contracting solution and the half-lines corresponding to $\vartheta=0$ and $\vartheta=\beta$ can be computed using the divergence theorem and the fact that $\kappa=\langle(x, u), \nu\rangle$. We obtain

$$
A=\frac{1}{2} \int_{\gamma} \kappa d \mu=\frac{1}{2}\left(2 \pi-\left(\pi-\frac{\pi}{3}\right)-(\pi-\beta)-\left(\pi-\frac{\pi}{2}\right)\right)=\frac{\beta}{2}-\frac{\pi}{12},
$$

where $\gamma$ denotes the curved part of the boundary of the domain and $d \mu$ is the corresponding volume element.

Recall from Figure 4 that for every fixed $\beta, b \leq b_{u}$ and $h \leq h_{\max }$ there is a natural common polygon (in dotted lines) bounding from above the area of the self-similar curve. A simple computation shows that the area of the quadrilateral 
$A_{\square}(\beta)$ is given by

$$
\begin{aligned}
A_{\square}(\beta) & =\frac{b_{u}^{2} \cos \beta \sin \beta}{2}+\left(h_{\max }-b_{u} \cos \beta\right) b_{u} \sin \beta-\frac{\left(h_{\max }-b_{u} \cos \beta\right)^{2} \tan \alpha}{2} \\
& =\frac{b_{u}^{2}}{4} \sin 2 \beta+\left(h_{\max }-b_{u} \cos \beta\right) b_{u} \sin \beta-\frac{\left(h_{\max }-b_{u} \cos \beta\right)^{2} \tan \alpha}{2},
\end{aligned}
$$

where $\alpha=\pi-\frac{\pi}{3}-\beta=\frac{2 \pi}{3}-\beta$. Furthermore, Proposition 5.6 implies that

$$
\frac{d A_{\square}}{d \beta}-\frac{d A}{d \beta}<0 .
$$

Hence an explicit calculation shows that

$$
\left(A_{\square}-A\right)(\beta) \leq\left(A_{\square}-A\right)\left(\pi-\frac{\pi}{\sqrt{2}}\right)<0 .
$$

5.2.2. Integral estimates. In order to finish the proof of uniqueness we use the support function. Recall that this is defined to be the map $S: S^{1} \times[0, T) \rightarrow \mathbb{R}$ given by

$$
S(x, t)=\left\langle x, F\left(\nu^{-1}(x), t\right)\right\rangle,
$$

where $\nu$ is the function $G \rightarrow S^{1}$ giving the unit normal vector to the curve. Note that $F\left(\nu^{-1}(x), t\right)$ is the point of the curve where $x$ is the unit normal vector. Furthermore $S^{1}$ is parameterized over its arc-length $\vartheta$, such that we get $S$ as a function of $(\vartheta, t) \in[0,2 \pi] \times[0, T)$. In general, the support function and the curvature are related by the formula

$$
\kappa^{-1}=S_{\vartheta \vartheta}+S .
$$

Hence, for self-similar shrinking solutions one has

$$
S(\vartheta)=\kappa(\vartheta)
$$

and the equation for the support function reads:

$$
S_{\vartheta \vartheta}+S=\frac{1}{S}
$$

In the following we will assume that $S>0$. An easy calculation shows that the energy $E^{S}$, defined as

$$
E^{S}\left(S, S_{\vartheta}\right):=\left(S_{\vartheta}\right)^{2}+S^{2}-2 \log S,
$$

is a first integral of the Equation (5.7). Therefore, each solution $S(\vartheta)$ of equation (5.7) lies on a level set of $E^{S}$. Note that this energy is equivalent to the energy considered in Lemma 5.3 since $-2 \log E^{u}=E^{S}$.

Let us have a closer look at the energy levels in the $\left(S, S_{\vartheta}\right)$-plane: $E^{S}$ has a unique critical point at $(1,0)$. The fact that

$$
D^{2} E^{S}(S, R)=\left(\begin{array}{cc}
2+\frac{2}{S^{2}} & 0 \\
0 & 2
\end{array}\right)
$$

is positive definite implies that the other level sets are convex closed curves around $(1,0)$. See also the energy diagram picture (Figure 5 ). 


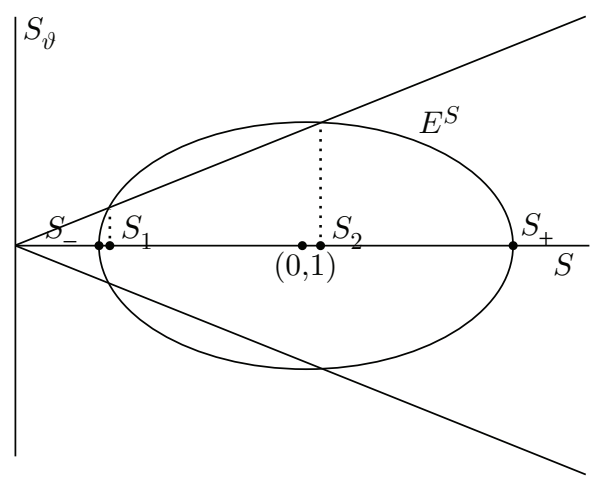

Figure 5. Energy diagram.

Furthermore, it follows that

- $E^{S}(S, R)>0$ for all $S>0$,

- $E^{S}(1,0)=1$,

- $E^{S}(S, 0) \longrightarrow \infty$ for $S \longrightarrow 0$ or $S \longrightarrow \infty$.

In order to impose the angle condition at the triple points we compute the points of each energy level where the embedding vector $X$ and the tangent vector $X_{\vartheta}$ form an angle of $\pm \frac{2 \pi}{3}$ or $\pm \frac{\pi}{3}$. (Later we will consider integrals that start at a point corresponding to a minimum of $|F|$. Thus we exclude the undesired angles.) This condition is equivalent to

$$
\frac{1}{2}=\left|\cos \frac{\pi}{3}\right|=\left|\cos \frac{2 \pi}{3}\right|=\frac{\left|\left\langle X, X_{\vartheta}\right\rangle\right|}{|X|\left|X_{\vartheta}\right|} .
$$

Now let us express $X$ and $X_{\vartheta}$ in terms of $S$ and $S_{\vartheta}$ :

$$
\begin{aligned}
X & =S \nu+S_{\vartheta} \nu_{\vartheta}, \\
X_{\vartheta} & =S \nu_{\vartheta}+S_{\vartheta \vartheta} \nu_{\vartheta} .
\end{aligned}
$$

Plugging these formulas into (5.9) leads to

$$
\frac{1}{2}=\frac{\left|S_{\vartheta}\right|}{\sqrt{S^{2}+S_{\vartheta}^{2}}}
$$

which is equivalent to

$$
S= \pm \sqrt{3} S_{\vartheta}
$$

Therefore, in terms of the energy diagram, a self-similar lens can be described as follows. It belongs to some energy level $E^{S}$ and the points corresponding to $(a(t), 0)$ and $(b(t), 0)$ lie on the lines $S=\sqrt{3} S_{\vartheta}$ and $S=-\sqrt{3} S_{\vartheta}$, respectively. Moreover, these endpoints correspond to some values $\vartheta_{a}$ and $\vartheta_{b}$ in the parameterization for the support function that satisfy $\vartheta_{a}-\vartheta_{b}=\frac{2 \pi}{3}$.

In the coming proof we make use of an analog of Ben Andrews' formula [2, p. $447,1.1$ ] that allows us to compute the total curvature between intersection points of a given energy level $E^{S}$ with the two half-lines $S= \pm \sqrt{3} S_{\vartheta}$. In particular we prove that the fact that the total curvature along the self-similar shrinking curve should be $\frac{2 \pi}{3}$ uniquely selects $E^{S}$ and the lens.

For a fixed energy level $E^{S}$, let us define $S_{-}$and $S_{+}$to be the left and right intersections of the energy level $E^{S}$ with the $S$-axis. Let $S_{1}$ and $S_{2}$ be the abscissas 
of the two intersections of that energy level of $E^{S}$ with the lines $S= \pm \sqrt{3} S_{\vartheta}$ if they exist (see also the energy diagram). Then, the integral of the curvature of the self-similar solution from $S_{-}$to $S_{1,2}$ is given by

$$
\Psi\left(S_{-}, S_{1,2}, E^{S}\right)=\int_{S_{-}}^{S_{1,2}} \frac{1}{\sqrt{E^{S}-S^{2}+2 \log S}} d S .
$$

For $S_{1} \leq S_{2}$, the quantity

$$
\tilde{\eta}=\frac{S_{2}}{S_{-}}
$$

parameterizes the energy levels (or at least those for which we have intersections with the half-lines, which are of interest). We will see that this also applies for $\bar{\eta}=\frac{S_{1}}{S_{-}}$. Set $x=\frac{S}{S_{-}}$and

$$
\Psi(\eta)=\int_{1}^{\eta} \frac{1}{\sqrt{1-x^{2}+\frac{4 \eta^{2}-3}{3 \log \eta} \log x}} d x .
$$

Thus, the total curvature of a symmetric lens is given by $2 \Psi(\eta)$. Similarly, for asymmetric lenses the total curvature can be computed by $\Sigma(\eta)=\Psi(\eta)+\Psi(\bar{\eta})$. (According to [2] none of our self-similarly shrinking lenses contains a point corresponding to $S_{+}$. This ensures in particular that the intersection angles are as desired.)

From the energy conservation we notice that (for a fixed lens) $\tilde{\eta}$ and $\bar{\eta}$, defined as above, satisfy the following relation:

$$
\frac{4 \bar{\eta}^{2}-3}{3 \log \bar{\eta}}=\frac{4 \tilde{\eta}^{2}-3}{3 \log \tilde{\eta}} .
$$

The expressions involved in this relation satisfy the following monotonicity property:

Lemma 5.7. The function

$$
(1, \infty) \ni \eta \mapsto \frac{1}{2} \cdot \frac{4 \eta^{2}-3}{3 \log \eta}
$$

is strictly convex, strictly decreasing near 1 and strictly increasing near $\infty$.

Proof. Observe that the derivative of this function is given by $A(\eta)$ as introduced below; see (5.15). Lemma 5.10 (ii) yields that $A^{\prime}<0$. Observe also that $A<0$ near 1 and $A>0$ near $\infty$.

Hence we deduce the following. For each $\tilde{\eta}$ there is at most one $\bar{\eta} \neq \tilde{\eta}$ such that (5.12) is satisfied and, thus, we can consider $\bar{\eta}$ as a function of $\tilde{\eta}$. Moreover, there is a unique $\eta_{0}$ such that $\bar{\eta}\left(\eta_{0}\right)=\eta_{0}$ and the function $\bar{\eta}(\tilde{\eta})$ is decreasing for $\tilde{\eta} \geq \eta_{0}$. Hence, $\bar{\eta} \leq \eta_{0}$ can also be considered as a function of $\tilde{\eta} \geq \eta_{0}$.

In this section we will use the following notation conventions. When we use the variable $\eta$, we refer to $\eta \in[1, \infty)$. If we restrict the values of $\eta$ to $\eta \geq \eta_{0}$, we will denote the variable by $\tilde{\eta}$. We will always assume that $1 \leq \bar{\eta} \leq \eta_{0} \leq \tilde{\eta}$ and we consider $\bar{\eta}$ as a function of $\tilde{\eta}$.

We want to remark that the above considerations imply that $\Sigma$ is a function of $\eta$ and that it is enough to study $\Sigma(\tilde{\eta})$ where $\tilde{\eta} \geq \eta_{0}$. 
Since the total curvature of a self-similarly shrinking solution is $\frac{2 \pi}{3}$, our uniqueness result follows from

Proposition 5.8. $\quad$ (i) The function $\Psi(\eta)$ attains the value $\frac{\pi}{3}$ at a unique point $\eta \in[1, \infty)$.

(ii) $\Sigma(\tilde{\eta})<\frac{2 \pi}{3}$ for every $\tilde{\eta} \geq \eta_{0}$.

Moreover, we can draw the following connection with the previous subsection:

Lemma 5.9. For $\eta \geq 1.9$ we have $\Psi(\eta) \neq \frac{\pi}{3}$ and $\Sigma(\tilde{\eta}) \neq \frac{2 \pi}{3}$.

Proof. Assume that $\Psi(\eta)=\frac{\pi}{3}$ or $\Sigma(\tilde{\eta})=\frac{2 \pi}{3}$. Then this $\eta$ (or correspondingly $\tilde{\eta})$ determines a self-similarly shrinking solution with correct intersection angles. Recall that $\tilde{\eta}$ denotes the same quantity as $\eta$ does, but restricted to $\left[\eta_{0}, \infty\right)$. Hence in the computations of this proof we will always refer to $\eta$, but it can be directly replaced by $\tilde{\eta}$ to obtain the desired conclusion for $\Sigma(\tilde{\eta})$.

By the definition of $h$ we have $S_{-}=h$ and $\eta=S_{1} / h$. At the point where our solution makes an angle of $\pi / 3$ with the ray from the origin, we get $S_{\vartheta}=1 / \sqrt{3} S=$ $1 / \sqrt{3} S_{1}$. As the energy is conserved we have

$$
h^{2}-2 \log h=E^{S}=S_{\vartheta}^{2}+S^{2}-2 \log S=\frac{4}{3} S_{1}^{2}-2 \log S_{1} .
$$

Rearranging terms gives

$$
2 \log \eta=\left(\frac{4}{3} \eta^{2}-1\right) h^{2} .
$$

From Proposition 5.5, it suffices to consider $h \geq h_{\max }$. Hence

$$
2 \log \eta \geq\left(\frac{4}{3} \eta^{2}-1\right) h_{\max }^{2} .
$$

It is easy to check that for $\eta \geq 1.9$, inequality (5.13) is violated.

The previous lemma implies that in order to prove Proposition 5.8 it suffices to focus on $1<\eta \leq 1.9$. In this range we perform a careful analysis of the functions $\Psi, \Sigma$ and their derivatives. We first compute

$$
\frac{d \Psi}{d \eta}(\eta)=\frac{\sqrt{3}}{\eta}-A(\eta) \int_{1}^{\eta} \frac{\log x}{\left(1-x^{2}+\frac{4 \eta^{2}-3}{3 \log \eta} \log x\right)^{\frac{3}{2}}} d x
$$

with

$$
A(\eta):=\frac{8 \eta^{2} \log \eta-4 \eta^{2}+3}{6 \eta \log ^{2} \eta} .
$$

Thus, $\Psi$ is increasing when $A(\eta) \leq 0$. In order to relate this computation to the analogous one for the function $\Sigma$, from (5.12) we notice

$$
A(\tilde{\eta})=A(\bar{\eta}) \frac{d \bar{\eta}}{d \tilde{\eta}}
$$

Prior to the proof of Proposition 5.8 we need a few elementary lemmata.

Lemma 5.10. The following estimates hold.

(i) $\frac{d A}{d \eta}(\eta) \equiv A^{\prime}(\eta)>0$ for all $\eta>1$. In particular, there is a unique $\eta_{0} \in[1, \infty)$ such that $A<0$ on $\left[1, \eta_{0}\right)$ and $A>0$ on $\left(\eta_{0}, \infty\right)$. Notice that this agrees with the previous definition of $\eta_{0}$. 
(ii) $\frac{\sqrt{3} \eta}{3 A(\eta) \log \eta}$ is decreasing for $\tilde{\eta}=\eta>1$ such that $A(\eta)>0$ and $\eta \leq e$ (in particular for $\eta \leq 1.9$ ).

(iii) The function $\eta \mapsto\left[1+\frac{4 \eta_{0}^{2}-3}{4 \eta^{2}-3}\right] \log \eta$ is increasing for $\eta \geq 1$.

Proof. (i) Define

$$
B(\eta):=\left(6 \eta^{2} \log ^{3} \eta\right) \frac{d A}{d \eta}(\eta)=8 \eta^{2} \log ^{2} \eta-12 \eta^{2} \log \eta-3 \log \eta+8 \eta^{2}-6 .
$$

We show that $B$ stays positive for all $\eta \geq 1$. This follows from $B(1)=2>0$ and $\frac{d B}{d \eta} \geq 0$ :

$$
\begin{aligned}
\frac{d B}{d \eta}(\eta) & =16 \eta \log ^{2} \eta-8 \eta \log \eta+4 \eta-\frac{3}{\eta} \\
& =\frac{1}{\eta}\left((4 \eta \log \eta-\eta)^{2}+3\left(\eta^{2}-1\right)\right) \geq 0 .
\end{aligned}
$$

(ii) By direct calculation, we obtain

$$
\frac{d}{d \eta} \frac{\sqrt{3} \eta}{3 A(\eta) \log \eta}=-\frac{\sqrt{3} A^{\prime}(\eta) \eta}{3 A^{2}(\eta) \log \eta}+\frac{\sqrt{3}}{3 A(\eta) \log ^{2} \eta}(\log \eta-1) .
$$

From (ii) the first term is negative. Since $A(\eta)>0$, the second term is negative for $\log \eta-1 \leq 0$ or equivalently $\eta \leq e$.

(iii) Set $c=4 \eta_{0}^{2}-3$. Taking a derivative we have

$$
\begin{aligned}
\frac{d}{d \eta}\left(\left[1+\frac{c}{4 \eta^{2}-3}\right] \log \eta\right) & =\frac{\eta}{\left(4 \eta^{2}-3\right)^{2}}\left[\left(4 \eta-\frac{3}{\eta}\right)^{2}+4 c-\frac{3 c}{\eta^{2}}-8 c \log \eta\right] \\
& \geq \frac{\eta}{\left(4 \eta^{2}-3\right)^{2}}\left[\left(4 \eta-\frac{3}{\eta}\right)^{2}+16-\frac{15}{\eta^{2}}-20 \log \eta^{2}\right] .
\end{aligned}
$$

Here we have used that $A(1.33652)>0, A(1.3365)<0$, hence $1.33652>\eta_{0}>$ 1.3365 and $5>c>4$. Taking derivatives, a direct computation implies that the last square bracket above is increasing. Since at $\eta=1$ this bracket is positive, we conclude the result.

Lemma 5.11. (i)

$$
\frac{\log x}{1-x^{2}+\frac{4 \eta^{2}-3}{3 \log \eta} \log x} \leq \frac{3 \log \eta}{\eta^{2}}
$$

for all $1 \leq x \leq \eta$ with strict inequality for $x<\eta$.

(ii) The function

$$
\frac{x-1}{1-x^{2}+\frac{4 \eta^{2}-3}{3 \log \eta} \log x} \equiv \frac{x-1}{d(x)}
$$

is an increasing function of $x$, for $\eta \geq x \geq 1$.

Proof. (i) The statement of the lemma is equivalent to $\frac{x^{2}-1}{\log x} \leq \frac{\eta^{2}-1}{\log \eta}$ for all $1 \leq$ $x \leq \eta$. Applying l'Hôpital's rule we see that $\frac{x^{2}-1}{\log x} \equiv \frac{f(x)}{g(x)} \rightarrow 2$ as $x \rightarrow 1$. The functions $x^{2}-1$ and $\log x$ are both positive for $x>1$. Consider $f^{\prime} g-g^{\prime} f$. It vanishes at $x=1$ and is positive for $x>0$ as $x^{2}-1$ is convex and $\log x$ is concave. Therefore the quotient is strictly increasing. 
(ii) Notice that

$$
\frac{x-1}{d(x)}=\frac{1}{-(1+x)+\frac{4 \eta^{2}-3}{3 \log \eta} \frac{\log x}{x-1}}
$$

and

$$
\begin{aligned}
\frac{d}{d x}\left(-(1+x)+\frac{4 \eta^{2}-3}{3 \log \eta} \frac{\log x}{x-1}\right) & =-1+\frac{4 \eta^{2}-3}{3 \log \eta}\left(\frac{1}{x(x-1)}-\frac{\log x}{(x-1)^{2}}\right) \\
& =-1+\frac{4 \eta^{2}-3}{3 \log \eta} \frac{1}{(x-1)^{2}}\left(1-\frac{1}{x}-\log x\right) \\
& \leq \frac{4 \eta^{2}-3}{3 \log \eta} \frac{1}{(x-1)^{2}}\left(1-\frac{1}{x}-\log x\right) .
\end{aligned}
$$

Since

$$
\frac{d}{d x}\left(1-\frac{1}{x}-\log x\right)=\frac{1}{x^{2}}-\frac{1}{x} \leq 0 \text { for } x>1,
$$

we conclude

$$
\frac{d}{d x}\left(-(1+x)+\frac{4 \eta^{2}-3}{3 \log \eta} \frac{\log x}{x-1}\right) \leq \frac{4 \eta^{2}-3}{3 \log \eta} \frac{1}{(x-1)^{2}}\left(1-\frac{1}{1}-\log (1)\right)=0 .
$$

Proof of Proposition 5.8. We prove (ii) and (iii) of the statement of Proposition 5.8 separately, interrupted by a lemma.

Proof of (ii). We divide the proof into the following steps.

(a) We start by observing $\frac{d \Psi}{d \eta}>0$ for $1<\eta \leq \eta_{0}$.

(b) We show $\Psi>\frac{\pi}{3}$ at critical points of $\Psi$ in the interval $[1,1.9)$.

(c) We prove $\Psi(1.9)>\frac{\pi}{3}$.

(d) We conclude the result from the previous steps.

Claim (a) follows directly from (5.14) and Lemma 5.10 (ii).

For claim (b), it suffices to consider $\eta>\eta_{0}$ as $\Psi$ is strictly increasing otherwise. Using Lemma 5.11 (ii), we estimate

$$
\begin{aligned}
\frac{d \Psi}{d \eta}(\eta) & >\frac{\sqrt{3}}{\eta}-A(\eta) \frac{3 \log \eta}{\eta^{2}} \int_{1}^{\eta} \frac{1}{\left(1-x^{2}+\frac{4 \eta^{2}-3}{3 \log \eta} \log x\right)^{\frac{1}{2}}} d x \\
& =\frac{\sqrt{3}}{\eta}-A(\eta) \frac{3 \log \eta}{\eta^{2}} \Psi(\eta) .
\end{aligned}
$$

Suppose that $\Psi$ has a critical point for some $\eta \in[1,1.9) \cap\left(\eta_{0}, 1.9\right)$. Then $\frac{d \Psi}{d \eta}(\eta)=$ 0 and thus

Lemma 5.10 (iii) implies that

$$
\Psi(\eta)>\frac{\sqrt{3} \eta}{3 A(\eta) \log \eta} .
$$

$$
\Psi(\eta)>\frac{\sqrt{3} \cdot 1.9}{3 A(1.9) \log 1.9} \geq \frac{\pi}{3},
$$

proving (b). 
In order to prove claim (IC), we use claim (Bb) and (5.17). According to Lemma 5.9 and Proposition 5.1. there exists $\eta<1.9$, such that $\Psi(\eta)=\frac{\pi}{3}$. Suppose that $\Psi(1.9) \leq \frac{\pi}{3}$. Then from (b),$\frac{d \Psi}{d \eta}(1.9) \leq 0$. However, from equation (5.17), we get $\frac{d \Psi}{d \eta}(1.9)>0$, which yields a contradiction.

Combining the previous steps, Proposition 5.1 and Lemma 5.9, (d) follows.

We need the following useful lemma for the proof of (iii).

\section{Lemma 5.12.}

$$
0.72<\Psi\left(\eta_{0}\right) \leq 0.785
$$

Proof. It is easy to check that $A(1.33652)>0$; therefore $\eta_{0} \leq 1.33652$. Moreover, if $\Psi(1.33652) \leq \frac{\pi}{3}$, the proof of Proposition 5.8 (ii) implies that $\Psi(\eta)$ is increasing for $\eta \leq 1.33652$ and $\Psi\left(\eta_{0}\right) \leq \Psi(1.33652)$. Hence, it is enough to show that

$$
\Psi(1.33652) \leq 0.785<\frac{\pi}{3} .
$$

Set $\delta x:=\frac{1.33652-1}{3}$. Using Lemma 5.11 (iii) we have

$$
\begin{aligned}
\Psi(1.33652) & \leq \int_{1}^{1+3 \delta x} \sqrt{\frac{x-1}{d(x)}} \sqrt{\frac{1}{x-1}} d x \\
& \leq \sum_{i=0}^{2} \int_{1+i \delta x}^{1+(i+1) \delta x} \sqrt{\frac{(i+1) \delta x}{d(1+(i+1) \delta x)}} \sqrt{\frac{1}{x-1}} d x \\
& =\sum_{i=0}^{2} \sqrt{\frac{(i+1) \delta x}{d(1+(i+1) \delta x)}} 2(\sqrt{(i+1) \delta x}-\sqrt{i \delta x}) \\
& \leq 0.785 .
\end{aligned}
$$

The lower bound can be computed analogously by noticing that $A(1.3365)<0$ and, thus, $\eta_{0}>1.3365$. We remark that as $x \searrow 1$ we consider the corresponding limit.

Proof of (iii). Let

$$
f(\tilde{\eta})=\Sigma(\tilde{\eta})-\sqrt{3} \log (\tilde{\eta} \bar{\eta})
$$

Using (5.16), we see that

$$
\begin{aligned}
& \frac{d f}{d \tilde{\eta}}=\frac{d \Sigma}{d \tilde{\eta}}-\frac{\sqrt{3}}{\tilde{\eta}}-\frac{\sqrt{3}}{\bar{\eta}} \frac{d \bar{\eta}}{d \tilde{\eta}} \\
&=-A(\tilde{\eta})\left(\int_{1}^{\tilde{\eta}} \frac{\log x}{\left(1-x^{2}+\frac{4 \tilde{\eta}^{2}-3}{3 \log \tilde{\eta}} \log x\right)^{\frac{3}{2}}} d x\right. \\
&\left.+\int_{1}^{\bar{\eta}} \frac{\log x}{\left(1-x^{2}+\frac{4 \tilde{\eta}^{2}-3}{3 \log \tilde{\eta}} \log x\right)^{\frac{3}{2}}} d x\right) \leq 0
\end{aligned}
$$

as $\tilde{\eta} \geq \eta_{0} \geq \bar{\eta}$. Therefore,

$$
\Sigma(\tilde{\eta}) \leq \Sigma\left(\eta_{0}\right)-\sqrt{3} \log \left(\eta_{0}^{2}\right)+\sqrt{3} \log (\tilde{\eta} \bar{\eta}) .
$$


Using Lemma 5.12 and that $\eta_{0}>1.3365$ we get

$$
\Sigma(\tilde{\eta}) \leq 2 \times 0.785-\sqrt{3} \log \left(1.3365^{2}\right)+\sqrt{3} \log (\tilde{\eta} \bar{\eta}) .
$$

From (5.12) observe that

$$
\log \bar{\eta}=\frac{4 \bar{\eta}^{2}-3}{4 \tilde{\eta}^{2}-3} \log \tilde{\eta} \leq \frac{4 \eta_{0}^{2}-3}{4 \tilde{\eta}^{2}-3} \log \tilde{\eta} .
$$

Hence, Lemma 5.10 (iii) implies for $\eta_{0} \leq \tilde{\eta} \leq 1.9$ that

$$
\begin{aligned}
\Sigma(\tilde{\eta}) & \leq \sqrt{3}\left[1+\frac{4 \eta_{0}^{2}-3}{4 \tilde{\eta}^{2}-3}\right] \log \tilde{\eta}+0.5653 \\
& \leq \sqrt{3}\left[1+\frac{4(1.33652)^{2}-3}{4(1.9)^{2}-3}\right] \log (1.9)+0.5653 \\
& <\frac{2 \pi}{3} .
\end{aligned}
$$

\section{Appendix A. Existence and uniqueness \\ OF HOMOTHETICALLY SHRINKING FISH-SHAPED NETWORKS}

We aim to prove the existence and uniqueness of a fish-shaped self-similarly shrinking network. By uniqueness we mean that up to rotations and reflections, this is the only self-similarly shrinking solution with two half-lines going to infinity, where the half-lines are not parallel and the network is topologically a lens.

We generalize the class of networks in which we are working. The name of the following class of networks is inspired by Theorem A.2 and Figure 2 .

Definition A.1 (Generalized lens-shaped networks). A generalized lens-shaped network is an embedding $F: G \rightarrow M \subset \mathbb{R}^{2}$, where $G$ is an abstract graph as in the definition of a lens-shaped network. We impose the following conditions on $F$ :

(i) $F$ is a homeomorphism on its image and, restricted to each edge, a diffeomorphism.

(ii) At the vertices the images of the edges meet at $120^{\circ}$.

(iii) When approaching infinity, the non-compact edges are close to straight halflines.

Assume that a self-similarly shrinking network contains a curve which is close to a half-line when approaching infinity. Then it is easy to see that this part of the network has to be a straight half-line.

Theorem A.2. There exists a family $\left(M_{t}\right)_{t \in(-\infty, 0)}$ of generalized lens-shaped networks that has the following properties:

(i) The networks $\left(M_{t}\right)_{t}$ shrink homothetically under (1.1) away from the triple points.

(ii) The bounded components of $\left(\mathbb{R}^{2} \backslash M_{t}\right)_{t}$ shrink to the origin in Hausdorff distance as $t \nearrow 0$.

(iii) The family $\left(M_{t}\right)_{t}$ is different from the one in Theorem 1.2

Moreover, up to rotations, $\left(M_{t}\right)_{t}$ is unique. We call such a network $M_{t}$ fish-shaped.

Proof. We distinguish whether the network is reflection symmetric with respect to an axis or not. Notice that results of Jörg Hättenschweiler [10, Lemma 3.17] imply 
that the network has such a symmetry. However, for the reader's convenience, we give an independent non-existence proof in the "asymmetric case".

Existence and uniqueness in the symmetric case. Note that the total curvature $K$ of the loop is given by

$$
K=\int_{\text {loop }} \kappa d s=2 \pi-\frac{2}{3} \pi=\frac{4 \pi}{3} .
$$

Note furthermore that the energy as defined in (5.4) is constant along the loop. At triple points this follows from the fact that at each triple point there is a straight half-line about which the network is locally symmetric. Thus both arcs which form the loop are part of the same self-similarly shrinking solution (without triple junctions). From the angle condition at the triple points there has to be at least one point of least distance $r_{\min }$ to the origin on each arc. As curved parts locally solve (5.2), 2. implies that local and global extrema of the distance to the origin coincide. Let us first assume that both arcs have only one point of least distance to the origin (and thus no point of maximal distance $r_{\max }$ to the origin). Since the distance to the origin is monotonically increasing between the point of minimal distance to the point of maximal distance, the only such solution is the one described in Theorem 1.2, where the two half-lines are parallel.

By Ben Andrews' results [2], the amount of curvature between $r_{\min }$ and $r_{\max }$ is at least $\pi / 2$. Thus the only possibility (besides a network as in Theorem 1.2) to form such a loop is that on the shorter arc we have only one minimum point and on the longer arc we have two minima and one maximum. Let us parameterize this family of solutions by $r_{\text {min }}$. Note that for a given $r_{\text {min }}$, there are in general two corresponding values $\bar{\eta} \leq \eta_{0}$ and $\tilde{\eta} \geq \eta_{0}$. To show existence and uniqueness we only have to show that the amount of the total curvature $K\left(r_{\text {min }}\right)$ attains the value $\frac{4 \pi}{3}$ only once. To do this write

$$
K\left(r_{\min }\right)=2 \int_{r_{\min }}^{r_{\max }} \kappa d s+4 \Psi(\eta) \equiv 2 \Theta(\rho)+4 \Psi(\eta),
$$

where $\eta=\eta\left(r_{\min }\right)$ and $\rho=r_{\max } / r_{\min }$. Ben Andrews has shown that $\lim _{\rho \rightarrow 1} \Theta(\rho)=$ $\frac{\pi}{\sqrt{2}}, \lim _{\rho \rightarrow \infty} \Theta(\rho)=\frac{\pi}{2}$ and that $\Theta$ is monotone in $\rho$; i.e., $\Theta\left(r_{\min }\right)$ is monotonically increasing in $r_{\min }$. Note also that in the interval [1, $\left.\eta_{0}\right], \bar{\eta}$ is monotonically increasing in $r_{\text {min }}$. From the proof of existence and uniqueness of the completely symmetric lens, we know that $\Psi(\eta)$ is strictly monotonically increasing in $\eta$ until it attains the value $\pi / 3$ and then it stays above this value. This yields that

$$
\lim _{r_{\min } \rightarrow 0} K\left(r_{\min }\right)=2 \lim _{\rho \rightarrow \infty} \Theta(\rho)=\pi .
$$

Restricting to $\bar{\eta} \leq \eta_{0}$, we see that $K\left(r_{\min }\right)$ is strictly increasing until $\bar{\eta}=\eta_{0}$. For $\bar{\eta}=\eta_{0}$ we have $K \geq \pi+4 \Psi\left(\eta_{0}\right) \geq \pi+4 \times 0.72>4 \pi / 3$. For $\tilde{\eta} \geq \eta_{0}, \Psi$ is increasing or bounded below by $\pi / 3$ and we obtain the same lower bound. Thus $K$ attains the value $4 \pi / 3$ exactly once. This implies existence and uniqueness in the symmetric case.

Non-existence in the asymmetric case. Assume that an asymmetric self-similarly shrinking generalized lens-shaped network exists, i.e. a network which has no axis of reflection. Let us suppose that the short arc is asymmetric, and thus also the 
long arc. Once more we can assume that we have the same configuration of minima and maxima as before. Let $\Sigma(\eta)$ be the total curvature of the short asymmetric arc. Using the considerations in Section 5 and Lemma 5.12 we see that

$$
\Sigma(\eta) \geq \Psi\left(\eta_{0}\right)>\frac{\pi}{6}
$$

Thus we get for the total curvature

$$
K\left(r_{\min }\right)=2 \Sigma(\eta)+2 \Theta(\rho)>\frac{\pi}{3}+\pi=\frac{4 \pi}{3},
$$

which yields a contradiction.

\section{NOTE ADDED IN PROOF}

We learned that Lemma 3.2 had been obtained in [15] and that several homothetic networks are classified in [3, Theorem 3].

\section{REFERENCES}

1. Uwe Abresch and Joel Langer, The normalized curve shortening flow and homothetic solutions, J. Differential Geom. 23 (1986), no. 2, 175-196. MR845704 (88d:53001)

2. Ben Andrews, Classification of limiting shapes for isotropic curve flows, J. Amer. Math. Soc. 16 (2003), no. 2, 443-459. MR.1949167 (2004a:53083)

3. Xinfu Chen and Jong-Shenq Guo, Self-similar solutions of a 2-D multiple-phase curvature flow, Phys. D 229 (2007), no. 1, 22-34. MR.2340176 (2008f:53087)

4. Julie Clutterbuck, Parabolic equations with continuous initial data, arXiv:math. AP/0504455.

5. Tobias H. Colding and William P. Minicozzi, II, Sharp estimates for mean curvature flow of graphs, J. Reine Angew. Math. 574 (2004), 187-195. MR.2099114 (2005g:53119)

6. Klaus Ecker and Gerhard Huisken, Mean curvature evolution of entire graphs, Ann. of Math. (2) 130 (1989), no. 3, 453-471. MR.1025164 (91c:53006)

7. Klaus Ecker and Gerhard Huisken, Interior estimates for hypersurfaces moving by mean curvature, Invent. Math. 105 (1991), no. 3, 547-569. MR1117150 (92i:53010)

8. Michael Gage and Richard S. Hamilton, The heat equation shrinking convex plane curves, J. Differential Geom. 23 (1986), no. 1, 69-96. MR840401 (87m:53003)

9. Matthew A. Grayson, The heat equation shrinks embedded plane curves to round points, J. Differential Geom. 26 (1987), no. 2, 285-314. MR906392 (89b:53005)

10. Jörg Hättenschweiler, Mean curvature flow of networks with triple junctions in the plane, Diplomarbeit, Zürich: ETH Zürich, Department of Mathematics, 46 pp., 2007.

11. Gerhard Huisken, Asymptotic behavior for singularities of the mean curvature flow, J. Differential Geom. 31 (1990), no. 1, 285-299. MR.1030675 (90m:53016)

12. Gerhard Huisken, A distance comparison principle for evolving curves, Asian J. Math. 2 (1998), no. 1, 127-133. MR1656553 (99m:58052)

13. Carlo Mantegazza, Matteo Novaga, and Vincenzo Maria Tortorelli, Motion by curvature of planar networks, Ann. Sc. Norm. Super. Pisa Cl. Sci. (5) 3 (2004), no. 2, 235-324. MR2075985 (2005d:53108)

14. William W. Mullins, Two-dimensional motion of idealized grain boundaries, J. Appl. Phys. 27 (1956), 900-904. MR0078836 (17:1252g)

15. John von Neumann, Discussion to 'Grain shapes and other metallurgical applications of topology' by C. S. Smith, Metal Interfaces, Amer. Soc. for Metals, Cleveland, Ohio, R. M. Brick (Editor), 1952, pp. 108-110. 
Department of Mathematics, Freie Universität Berlin, Arnimallee 3, 14195 Berlin, Germany

E-mail address: Oliver.Schnuerer@math.fu-berlin.de

Current address: Fachbereich Mathematik und Statistik, Universität Konstanz, 78457 Konstanz, Germany

E-mail address: Oliver.Schnuerer@uni-konstanz.de

Department of Mathematics, Freie Universität Berlin, Arnimallee 3, 14195 Berlin, Germany

E-mail address: azouani@math.fu-berlin.de

Department of Mathematics, Freie Universität Berlin, Arnimallee 3, 14195 Berlin, GERMAnY

E-mail address: georgi@math.fu-berlin.de

Department of Mathematics, Freie Universität Berlin, Arnimallee 3, 14195 Berlin, GERMANY

E-mail address: blanca@math.fu-berlin.de

Department of Mathematics, Freie Universität Berlin, Arnimallee 3, 14195 Berlin, GERMANY

E-mail address: jangle@math.fu-berlin.de

Mathematisches Institut der Eberhard-Karls-Universität Tübingen, Auf der MorGenstelle 10, D-72076 Tübingen, Germany

E-mail address: akoeller@everest.mathematik.uni-tuebingen.de

Department of Mathematics, Freie Universität Berlin, Arnimallee 3, 14195 Berlin, GERMAnY

E-mail address: marxen@math.fu-berlin.de

Department of Mathematics, Freie Universität Berlin, Arnimallee 3, 14195 Berlin, GERMANY

E-mail address: ritthale@math.fu-berlin.de

Max Planck Institute for Gravitational Physics (Albert Einstein Institute), WisSenschaftspark Golm, Am Mühlenberg 1, 14476 Golm, Germany

E-mail address: mariel@aei.mpg.de

Current address: Edificio Rolando Chuaqui, Facultad de Matemáticas, Pontificia Universidad Católica de Chile, Avda. Vicuña Mackenna 4860, Macul, Santiago, Chile

E-mail address: mariel@mat.puc.cl

Department of Mathematics, Freie Universität Berlin, Arnimallee 3, 14195 Berlin, Germany

E-mail address: Felix.Schulze@math.fu-berlin.de

Department of Mathematics, Freie Universität Berlin, Arnimallee 3, 14195 Berlin, GERMAnY

E-mail address: bsmith@math.fu-berlin.de 